\title{
Symmetries of Julia sets for analytic endomorphisms of the Riemann sphere
}

\author{
Gustavo Rodrigues Ferreira \\ DISSERTATION SUBMITTED \\ IN PARTIAL FULFILMENT OF THE \\ REQUIREMENTS FOR THE DEGREE OF \\ MASTER OF SCIENCE \\ AT THE \\ University of SÃo PAUlo \\ Programme: Applied Mathematics \\ Supervisor: Prof. Dr. Luciana Luna Anna Lomonaco
}

The author was financially supported by CNPq throughout the development of this work

São Paulo, August 2019 


\section{Symmetries of Julia sets for analytic endomorphisms of the Riemann sphere}

This version of the dissertation contains the corrections and alterations suggested by the Thesis Committee during the defence of this work's original version, on 25/07/2019. A copy of the original version is available at the Institute of Mathematics and Statistics of the University of São Paulo.

Thesis Committee

- Prof. Dr. Luciana Luna Anna Lomonaco (supervisor) - IME-USP

- Prof. Dr. Sylvain Philippe Pierre Bonnot - IME-USP

- Prof. Dr. Peter Edward Hazard - UFABC 
In memory of my father. 


\section{Acknowledgements}

There are so many people to thank that I barely know where to start. First of all, I am grateful to my friends for their support during the two long years of this project. A special thanks to Helder Nakaya and the folks at CSBL, who good-naturedly endured my continued presence at their lab!

I would also like to thank Mitsu Shishikura, Sebastian van Strien, Fedor Pakovic, Sylvain Bonnot and Laura DeMarco for their comments and suggestions to this work.

To my adviser, Luna Lomonaco, no thanks would ever be enough. For her patience, her advice, her support and her never-ending willingness to hear out my ramblings, I can only offer my deepest, deepest gratitude.

Finally, I want to thank my family for helping me become who I am. Words will never suffice. 


\section{Abstract}

FERREIRA, G. R. Symmetries of Julia sets for analytic endomorphisms of the Riemann sphere. 2019. 47 pages. Dissertation (MSc.) - Institute of Mathematics and Statistics, University of São Paulo, São Paulo, 2019.

Since the 1980s, much progress has been done in completely determining which functions share a Julia set. The polynomial case was completely solved in 1995, and it was shown that the symmetries of the Julia set play a central role in answering this question. The rational case remains open, but it was already shown to be much more complex than the polynomial one. In this thesis, we review existing results on rational maps sharing a Julia set, and offer results of our own on the symmetry group of such maps.

Keywords: Dynamical systems, holomorphic dynamics, symmetries. 


\section{Resumo}

FERREIRA, G. R. Simetrias de conjuntos de Julia para endomorfismos analíticos da esfera de Riemann. 2019. 47 p. Dissertação (Mestrado) - Instituto de Matemática e Estatística, Universidade de São Paulo, São Paulo, 2019.

Desde a década de oitenta, um enorme progresso foi feito no problema de determinar quais funções têm o mesmo conjunto de Julia. O caso polinomial foi completamente respondido em 1995, e mostrou-se que as simetrias do conjunto de Julia têm um papel central nessa questão. O caso racional permanece aberto, mas já se sabe que ele é muito mais complexo do que o polinomial. Nesta dissertação, nós revisamos resultados existentes sobre aplicações racionais com o mesmo conjunto de Julia e apresentamos nossos próprios resultados sobre o grupo de simetrias de tais aplicações.

Palavras-chave: Sistemas dinâmicos, dinâmica holomorfa, simetrias. 


\section{Contents}

List of Symbols $\quad$ vi

List of Figures $\quad$ vii

1 Introduction $\quad 1$

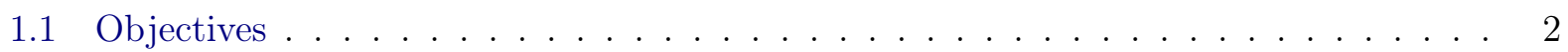

1.2 Organisation of this Work . . . . . . . . . . . . . . . 2

2 Preliminaries $\quad 3$

2.1 Holomorphic Dynamics . . . . . . . . . . . . . . . . . . . . . . . . . . . 3

2.2 Lie Groups . . . . . . . . . . . . . . . . . . . . . . . 6

2.3 Potential Theory . . . . . . . . . . . . . . . . . . . . . . 10

3 Previous Results $\quad 23$

3.1 Symmetries in Polynomial Dynamics . . . . . . . . . . . . . . . . 23

3.2 The Maximal Entropy Measure for Rational Functions . . . . . . . . . . . . . . . . . 29

3.3 A Modified Green's Function for Rational Maps . . . . . . . . . . . . . . . . . . . 32

4 Results $\quad 34$

4.1 General Considerations . . . . . . . . . . . . . . . . . 34

4.2 Applications . . . . . . . . . . . . . . . . . . . . . 41

5 Conclusions $\quad 45$

$\begin{array}{ll}\text { Bibliography } & 46\end{array}$ 


\section{List of Symbols}

$\begin{array}{ll}\mathcal{I}(X) & \text { The isometry group of the metric space } X \\ \widehat{\mathbb{C}} & \text { The Riemann sphere; } \mathbb{C} \cup\{\infty\} \\ P, Q & \text { Polynomials of degree } \geq 2 \\ R, S & \text { Rational maps of degree } \geq 2 \\ J(R), F(R) & \text { The Julia and Fatou sets for the map } R \\ \Sigma(R) & \text { The symmetry group of } J(R) \\ \mu_{R} & \text { The maximal entropy measure of } R \\ \Omega & \text { A domain of the Riemann sphere } \\ g_{\Omega}(z, w) & \text { The Green's function for } \Omega \text { with pole at } w \\ G, \mathfrak{g} & \text { The Lie group } G \text { and its associated Lie algebra } \mathfrak{g}\end{array}$




\section{List of Figures}

3.1 The figure shows the infamous Mandelbrot set, with highlights for the filled Julia sets of certain values of $c \ldots \ldots \ldots \ldots \ldots$

4.1 Above, the parameter plane for a family of singular perturbations with $m=d=2$. Below, Julia sets for particular values of $\lambda \ldots \ldots$. . . . . . . . . . . 43

4.2 Above, the parameter plane for a family of singular perturbations with $m=2$ and $d=1$. Below, Julia sets for particular values of $\lambda \ldots \ldots \ldots \ldots 4$ 


\section{Chapter 1}

\section{Introduction}

Given a space $X$ and a function $f: X \rightarrow X$, both with some prescribed structure, it can be said that the iterations of $f$ form a dynamical system. One is then interested in how the iterates affect points of the space; given $x \in X$, its orbit under $f$ is defined as $\mathcal{O}(x)=\left\{x, f(x), f^{2}(x), \ldots\right\}$ - here, $f^{n}$ denotes the n-fold composition of $f$ with itself.

In the last century, a particular case that has gathered attention is the study of holomorphic dynamics. Here, $X=\widehat{\mathbb{C}}=\mathbb{C} \cup\{\infty\}$, the Riemann sphere, and $f$ is some analytic self-map, or endomorphism, of $\widehat{\mathbb{C}}$. The interest in this setting comes from the fact that the dynamics of an endomorphism will, in many a case, result in a partition of the space: there is a set of points with "chaotic" behaviour, and its complement, where dynamics are "regular". While an in-depth discussion must wait until we have better definitions, we can look at a particular example.

Let $f(z)=z^{2}$. If $|z|<1$, then $\left|f^{n}(z)\right|=|z|^{2^{n}}$, which goes to zero as $n$ grows; at the same time, if $|z|>1$, then $f^{n}(z)$ eventually escapes to infinity. The points 0 and $\infty$ are unchanged by the action of $f$, making them fixed points. This is "regular" dynamics; nearby points behave in roughly the same way.

If $|z|=1$, however, the behaviour changes drastically. Writing $z=e^{i \theta}$, we can see that $f^{n}(z)=$ $e^{i 2^{n} \theta}$, and two facts become apparent: firstly, periodic points are dense in the unit circle. Indeed, periodic points of period $n$ are given by $2^{n} \theta=\theta$ modulo $2 \pi$, and the solutions to this are exactly the $\left(2^{n}-1\right)$-th roots of unity. Combining all values of $n$ gives us a set that is dense in the circle. Secondly, if $\theta$ is irrational, then $f^{n}(z)$ wanders throughout the circle without ever visiting the same point twice - giving us a dense orbit. Since irrational numbers are dense, the set of "wandering" points is also dense. This is what we mean by "chaotic" dynamics: every interval on the circle contains infinitely many periodic points, but also infinitely many points with dense orbits!

This partition of the space was first studied by Pierre Fatou (1878 - 1929) and Gaston Julia (1893 - 1978). Using tools from complex analysis, they proved that it happens for every rational function of degree greater than or equal to two. They also observed that most cases are not like the example given above: the set of chaotic dynamics often has a complex geometry. Thus, much attention has been given to this set - denoted the Julia set - in the study of complex dynamics.

One problem in particular concerns equality: when do two rational functions share the same Julia set? Julia himself proved that, if two rational functions commute, they share a Julia set [Jul22]. Later, in the 80s and 90s, this problem was completely solved for polynomials by the work of Baker, Eremenko, Beardon, Steinmetz and others. In the process, they discovered that this problem is intricately related to the symmetries of the Julia set [BE87, Bea90, SS95]; therefore, a lot of attention has been devoted to understanding and extending these results to general rational functions. Most remarkably, Ye [Ye15] proved that the classification scheme discovered by Schmidt and Steinmetz for polynomials fails for a non-negligible set of rational functions.

This is the context for this work; we tackle the problem of identifying the symmetries for Julia sets of rational functions. We offer partial extensions to Beardon's results for the polynomial case and apply them to a family of examples known as McMullen maps. 


\section{$1.1 \quad$ Objectives}

The initial aim of this dissertation is to understand the existing results on the symmetries of Julia sets for polynomials, proved by Emerenko, Beardon, Steinmetz and others (see Chapter 3). Then, we prove our own results for the symmetries of Julia sets of rational functions as partial extensions of Beardon's results.

\subsection{Organisation of this Work}

In Chapter 2, we introduce the necessary concepts from dynamical systems and Lie group theory. Chapter 3 reviews previous results on the symmetries of complex-analytic maps - both the polynomial case, which is completely solved, and the rational one, which is open. We move on to our own results for symmetries of rational functions in Chapter 4 and discuss our conclusions in Chapter 5. 


\section{Chapter 2}

\section{Preliminaries}

We divide this chapter in three sections, exposing the main results concerning holomorphic dynamics, Lie groups and potential theory. Any results we do not demonstrate are accompanied by a reference to a full proof.

\subsection{Holomorphic Dynamics}

In this section, we elucidate the concepts of Julia and Fatou sets, which we hinted at in the previous chapter, and expose the relevant result in the dynamics of rational maps on $\widehat{\mathbb{C}}$. The metric on the Riemann sphere is given by identifying it with $S^{2} \subset \mathbb{R}^{3}$ and taking the Riemannian metric induced by the Euclidean one in $\mathbb{R}^{3}$. We denote it the spherical metric; alternatively, we may consider the (equivalent) chordal metric defined as $\rho(z, w)=\|z-w\|_{\mathbb{R}^{3}}$.

Given an analytic map $R: \widehat{\mathbb{C}} \rightarrow \widehat{\mathbb{C}}$, we mentioned a fundamental dichotomy of complex dynamics, dividing the Riemann sphere into a set of chaotic dynamics, which we called the Julia set, and a set with "regular" dynamics. In order to explore their properties, we first make our definitions more precise. What we call "regular" dynamics shall be related to the behaviour of the iterates $\left\{R^{n}\right\}_{n \geq 1}$.

Definition. A family $\mathcal{F}=\left\{f_{\alpha}: U \subset \widehat{\mathbb{C}} \rightarrow \widehat{\mathbb{C}}\right\}_{\alpha \in A}$ of analytic functions is said to be normal if every sequence in $\mathcal{F}$ admits a subsequence that converges uniformly over compact subsets of $U$.

The following theorem shows that normal families encode what we understand by "regular" dynamics.

Theorem 2.1.1 (Arzelá-Ascoli). A family $\mathcal{F}=\left\{f_{\alpha}: U \subset \widehat{\mathbb{C}} \rightarrow \widehat{\mathbb{C}}\right\}_{\alpha \in A}$ of continuous functions is normal if, and only if, it is equicontinuous on every compact subset of $U$.

Proof. First, we prove the necessity of equicontinuity. Suppose by contradiction that $\mathcal{F}$ is normal but not equicontinuous on some compact subset $E$ of $U$. Choose sequences of points $z_{n}, z_{n}^{\prime} \in E$ and functions $f_{n} \in \mathcal{F}$ such that $\rho\left(z_{n}, z_{n}^{\prime}\right) \rightarrow 0$ but, at the same time, $\rho\left[f_{n}\left(z_{n}\right), f_{n}\left(z_{n}^{\prime}\right)\right] \geq \epsilon$ for all $n$. We can choose subsequences of $z_{n}$ and $z_{n}^{\prime}$ converging to a common limit $z^{\prime \prime}$, and also a subsequence of $f_{n}$ that converges uniformly over $E$ to some function $f$. It is important to remark that $f$, being a continuous function on a compact set, is uniformly continuous. Now, we choose the three subsequences so that they share the same subscripts $n_{k}$. For $\epsilon>0$, since $f_{n} \rightarrow f$ uniformly on $E$, we can choose $k$ such that $\rho\left[f_{n_{k}}\left(z_{n_{k}}\right), f\left(z_{n_{k}}\right)\right]<\epsilon / 3$, and at the same time $\rho\left[f_{n_{k}}\left(z_{n_{k}}^{\prime}\right), f\left(z_{n_{k}}^{\prime}\right)\right]<\epsilon / 3$. By the uniform continuity of $f, k$ can also be chosen so that $\rho\left[f\left(z_{n_{k}}\right), f\left(z_{n_{k}}^{\prime}\right)\right]<\epsilon / 3$, and so by the triangle inequality

$$
\rho\left[f_{n_{k}}\left(z_{n_{k}}\right), f_{n_{k}}\left(z_{n_{k}}^{\prime}\right)\right] \leq \rho\left[f_{n_{k}}\left(z_{n_{k}}\right), f\left(z_{n_{k}}\right)\right]+\rho\left[f_{n_{k}}\left(z_{n_{k}}^{\prime}\right), f\left(z_{n_{k}}^{\prime}\right)\right]+\rho\left[f\left(z_{n_{k}}\right), f\left(z_{n_{k}}^{\prime}\right)\right]<\epsilon,
$$

contradicting our original assumption.

For proving sufficiency, we proceed by a diagonal argument. First, we take a dense, countable set of points in $U$ - for instance, the points $q_{k} \in U$ with rational real and imaginary parts. Now, for 
a sequence $f_{n} \in \mathcal{F}$, we shall use the compacity of $\widehat{\mathbb{C}}$ to fabricate a subsequence of $f_{n}$ that converges on every point $q_{k}$. First, consider the sequence $f_{n}\left(q_{1}\right)$. Since $\widehat{\mathbb{C}}$ is compact, it admits a subsequence $f_{n_{1 j}}\left(q_{1}\right)$ that converges to some $\bar{f}_{1} \in \widehat{\mathbb{C}}$. Now, we take our subsequence $f_{n_{1 j}}$ and evaluate it at $q_{2}$. By the same argument, there exists a subsequence $f_{n_{2 j}}$ such that $f_{n_{2 j}}\left(q_{2}\right)$ converges to a point $\bar{f}_{2} \in \widehat{\mathbb{C}}$. Proceeding in the same manner for all $k$, we find an array of subscripts

$$
\begin{aligned}
& n_{11}<n_{12}<n_{13}<\cdots \\
& n_{21}<n_{22}<n_{23}<\cdots \\
& n_{31}<n_{32}<n_{33}<\cdots
\end{aligned}
$$

such that each line is contained in the previous one, and $f_{n_{k j}}\left(q_{k}\right) \rightarrow \bar{f}_{k} \in \widehat{\mathbb{C}}$ as $j \rightarrow \infty$ for every $k$. Choosing the diagonal subscripts in the array above, we find that the sequence $f_{n_{j j}}$ converges over every $q_{k} \in U$. For ease of notation, we replace $n_{j j}$ by $n_{j}$.

Now, for a compact subset $E \subset U$, we shall prove that $f_{n_{j}}$ converges uniformly on $E$. Since $\mathcal{F}$ is equicontiuous on $E$, given any $\epsilon>0$ we can choose $\delta>0$ such that, for $z, z^{\prime} \in E, \rho\left(z, z^{\prime}\right)<\delta$ implies $\rho\left[f(z), f\left(z^{\prime}\right)\right]<\epsilon / 3$ for every $f \in \mathcal{F}$. Since $E$ is compact, we can cover it by a finite number of balls of radius $\delta / 2$. Now, for any $z \in E, z$ is contained in such a ball and, since the $q_{k}$ are dense in $U$, there is also at least one point $q_{k}$ in the same ball - i.e., $\rho\left(z, q_{k}\right)<\delta$. Choosing one such point from each ball yields a finite collection $q_{l}$, and we can select an $i_{0}$ such that, for $i, j>i_{0}$, $\rho\left[f_{n_{i}}\left(q_{l}\right), f_{n_{j}}\left(q_{l}\right)\right]<\epsilon / 3$ for every $l$. Thus, by the triangle inequality,

$$
\rho\left[f_{n_{i}}(z), f_{n_{j}}(z)\right]<\rho\left[f_{n_{i}}(z), f_{n_{i}}\left(q_{l}\right)\right]+\rho\left[f_{n_{j}}(z), f_{n_{j}}\left(q_{l}\right)\right]+\rho\left[\left(f_{n_{i}}\left(q_{l}\right), f_{n_{j}}\left(q_{l}\right)\right]<\epsilon\right.
$$

for every $z$ in $E$, and it follows that $f_{n_{j}}$ converges uniformly over $E$.

For the family $\left\{R^{n}\right\}_{n \geq 1}$, this means that nearby points remain nearby under iteration of $R$ whenever $\left\{R^{n}\right\}_{n \geq 1}$ is normal, which implies a relatively tame behaviour of the dynamics. Where it is not, it can be proved that the dynamics are "chaotic" in the sense of Devaney [Dev03, MNTU00]. We have the following definition:

Definition. Let $R$ be a rational function on the Riemann sphere. The Fatou set of $R$, denoted $F(R)$ - or just $F$ if it will not lead to ambiguity -, is the maximal open set where $\left\{R^{n}\right\}_{n \geq 1}$ is a normal family. Its complement is called the Julia set of $R$ and denoted by $J(R)$.

This is the starting point for analysing the dynamics of rational functions. From our previous discussion, we see that $F(R)$ is what was previously called the set of "regular" dynamics. We start by proving general properties of the Julia set, and it is again apparent that normal families are a powerful tool in the study of complex dynamics.

Proposition 2.1.1. For $R: \widehat{\mathbb{C}} \rightarrow \widehat{\mathbb{C}}$ a rational map of degree $d \geq 2$, the set $J(R)$ satisfies:

(i) It is nonempty;

(ii) It is completely invariant, i.e., $R(J)=R^{-1}(J)=J$;

(iii) If $E$ is a closed, completely invariant subset of $\widehat{\mathbb{C}}$ with more than three points, then $J(R) \subset E$;

(iv) It contains the repelling periodic points of $R$.

Proof. (i) Suppose, by contradiction, that $J=\emptyset$. Then, by the definition of $F$, the sequence $R^{n}$ should admit some subsequence $R^{n_{j}}$ converging uniformly over $\widehat{\mathbb{C}}$ to some analytic $g: \widehat{\mathbb{C}} \rightarrow \widehat{\mathbb{C}}$. By Hurwitz's theorem, it must be the case that $\operatorname{deg}\left(R^{n_{j}}\right)=\operatorname{deg}(g)$ for all large enough $j$. However, it is also a fact that $\operatorname{deg}\left(R^{n_{j}}\right)=d^{n_{j}}$, which diverges to infinity. 
(ii) Since $J=\widehat{\mathbb{C}} \backslash F$, it is equivalent to prove that $F$ is completely invariant under $R$. Furthermore, as $R$ is surjective, it suffices to prove that $F$ is backward invariant, i.e., $R^{-1}(F)=F$. Thus, let $z_{0}$ be any point in $R^{-1}(F)$ and define $w_{0}=R\left(z_{0}\right)$, so that $w_{0} \in F(R)$. It follows that the family $\left\{R^{n}\right\}_{n \geq 1}$ is normal at $w_{0}$ and, by continuity of $R$, we conclude that $\left\{R^{n}\right\}_{n \geq 1}$ is normal at $z_{0}$ and $z_{0} \in F(R)$.

(iii) This is a direct consequence of Montel's theorem [Bea91], which states that a family $\left\{F_{\alpha}\right.$ : $U \rightarrow \widehat{\mathbb{C}}\}_{\alpha \in A}$ of holomorphic maps excluding three points in $\widehat{\mathbb{C}}-$ i.e., there exist $z_{1}, z_{2}, z_{3} \in \widehat{\mathbb{C}}$ such that $\left\{z_{1}, z_{2}, z_{3}\right\} \cap F_{\alpha}(U)=\emptyset$ for every $\alpha \in A$ - is normal. Indeed, if $J(R)$ is not a subset of $E$, then we take $z \in J(R) \backslash E$ and a neighbourhood $U$ of $z$ that does not intersect $E$ ( $U$ is guaranteed to exist, since $E$ is closed). As $E$ is completely invariant, it must be the case that

$$
\bigcup_{n \geq 1} R^{n}(U) \subset \widehat{\mathbb{C}} \backslash E .
$$

Since $E$ has more than three points, it follows that $\left\{R^{n}\right\}_{n \geq 1}$ is normal in $U$, which is a contradiction.

(iv) Consider $z$ a repelling fixed point of $R$ and assume by contradiction that $z \in F$. We know, then, that $\left\{R^{n}\right\}_{n \geq 1}$ is normal at $z$ and thus admits a converging subsequence $\left\{R^{n_{j}}\right\}$. By Weierstrass's theorem, $\left(R^{n_{j}}\right)^{\prime}$ also converges to some holomorphic function $g$. With $R^{\prime}(z)=\lambda$ such that $|\lambda|>1$, we conclude that $R^{n_{j}}(z)=z$ and $\left(R^{n_{j}}\right)^{\prime}(z)=\lambda^{n_{j}}$, which diverges to infinity. This contradicts our hypotheses of convergence to a holomorphic $g$.

This last statement could, in fact, be strengthened: $J$ is the closure of all repelling periodic points of $R$ [Bea91]. Also, (iii) is often stated as saying that $J(R)$ is the minimal closed, completely invariant set with more than three points.

We move on to the local behaviour of the map near fixed points. As with real-valued dynamical systems, we expect some kind of linearisation based on the derivative of $R$. In fact, several results are available depending on the absolute value of the derivative. Here, we state only those that shall be of use to us.

Theorem 2.1.2 (Koenig, Böttcher). Suppose $z_{0}$ is a fixed point of $R$ with multiplier $\lambda$. Then:

(i) If $0<|\lambda|<1$, there exist neighbourhoods $U$ of $z_{0}, V$ of 0 and a unique (up to multiplication by a non-zero constant) biholomorphic map $\Phi: U \rightarrow V$ such that $\Phi\left(z_{0}\right)=0$ and $\Phi[R(z)]=\lambda \Phi(z)$.

(ii) If $\lambda=0$, then there exist neighbourhoods $U$ and $V$ as above and a biholomorphic map $\Phi: U \rightarrow$ $V$ satisfying $\Phi\left(z_{0}\right)=0$ and $\Phi[R(z)]=[\Phi(z)]^{m}$, where $m>1$ is the local degree of $R$ at $z_{0}$.

Proof. We can conjugate $R$ by a translation, and thus assume that $z_{0}=0$.

We prove (i) first, and we start with uniqueness. If there were two such maps $\Phi$ and $\Psi$, their composition $\Psi \circ \Phi^{-1}$ would commute with the map $w \mapsto \lambda z$. Expanding as a power series,

$$
\Psi \circ \Phi^{-1}(w)=b_{1} w+b_{2} w^{2}+\cdots,
$$

and we can compose with multiplication by $\lambda$ on both the left and the right to conclude (comparing coefficients) that $\lambda b_{n}=b_{n} \lambda^{n}$ for all $n$. Since $\lambda$ is neither zero nor a root of unity, it follows that $b_{2}=b_{3}=\cdots=0$. Thus, $\Psi \circ \Phi^{-1}(w)=b_{1} w$, and $\Psi(z)=b_{1} \Phi(z)$.

For a proof of existence, we have by Taylor's theorem that there exist constants $C$ and $r_{0}$ such that

$$
|f(z)-\lambda z| \leq C\left|z^{2}\right| \quad \text { for } \quad|z|<r_{0} .
$$

Choose $c<1$ so that $c^{2}<|\lambda|<c$, and pick $0<r \leq r_{0}$ satisfying $|\lambda|+C r<c$. Whenever $|z|<r$, it follows that

$$
|f(z)| \leq|\lambda z|+C\left|z^{2}\right| \leq c|z| \text {. }
$$


Set $z_{n}=f^{n}(z)$; for $|z|<r$, the above expression implies that $\left|z_{n}\right| \leq r c^{n}$, and using Taylor's theorem again (with the same constants, since $r \leq r_{0}$ ) we obtain

$$
\left|z_{n+1}-\lambda z_{n}\right| \leq C\left|z_{n}\right|^{2} \leq C r^{2} c^{2 n} .
$$

Setting $w_{n}=z_{n} / \lambda^{n}$, we conclude that

$$
\left|w_{n+1}-w_{n}\right| \leq \frac{C r^{2}}{|\lambda|}\left(\frac{c^{2}}{|\lambda|}\right)^{n}
$$

Since $c<|\lambda|$, the right side of the inequality above goes to zero as $n \rightarrow \infty$, and thus the sequence $w_{n}$ converges uniformly over $|z|<r$ to a holomorphic limit $\Phi(z)=f^{n}(z) / \lambda^{n}$ (the uniformity of the convergence follows from the fact that the terms on the right side of the inequality do not depend on a particular choice of $z)$. The definition of $\Phi$ implies immediately that $\Phi[f(z)]=\lambda \Phi(z)$. Furthermore, by Weierstrass's convergence theorem, $\Phi^{\prime}(0)=1$ - since every term in the sequence $f^{n}(z) / \lambda^{n}$ has unit derivative at zero - and so $\Phi$ is locally a conformal isomorphism.

(ii) has a very similar proof, with $\Phi(z)$ defined as $\Phi(z)=\lim _{n \rightarrow \infty} \sqrt[n]{f^{n}(z)}$ for a well-defined choice of the $n$-th root. The details of the proof can be found in [Mil06a].

Among all holomorphic functions, polynomials are particularly rigid. It is only natural, then, to explore the consequences of this rigidity concerning their dynamical behaviour.

First and foremost, any polynomial $P$ fixes infinity; furthermore, its multiplier is zero, and thus all polynomials have a superattracting fixed point at infinity. Its immediate basin of attraction is denoted by $F_{\infty}$ - we recall that the complement of $F_{\infty}$ is $K(P)$, the filled Julia set. Since $P^{-1}\{\infty\}=\{\infty\}$, it must be the case that $F_{\infty}$ is connected, for any other connected component would necessarily contain a pre-image of infinity.

A crucial consequence of this simple fact is the following.

Proposition 2.1.2. For any polynomial $P: \mathbb{C} \rightarrow \mathbb{C}, J=\partial F_{\infty}$.

Proof. The domain $F_{\infty}$ is - from its definition - forward invariant; since $P$ does not have any poles, it follows that it is also backward invariant, and thus completely invariant. Therefore, $\overline{F_{\infty}}$ is a completely invariant closed set with more than three points. Thus, $J \subset \partial F_{\infty}$, and so $J=\partial F_{\infty}$.

\subsection{Lie Groups}

Roughly speaking, a Lie group is a group with the additional structure of a smooth manifold. They were first studied by the Norwegian mathematician Sophus Lie (1842 - 1899) at the end of the XIXth century, as he attempted to describe the way symmetry groups act on certain spaces. We shall work our way up to a precise definition, but we do assume some knowledge on differentiable manifolds.

Definition. A group is a non-empty set $G$ endowed with a binary operation $\cdot: G \times G \rightarrow G$, often denoted by juxtaposition, that satisfies the following axioms.

(i) $G$ is closed under ·, i.e., $x, y \in G$ implies that $x y \in G$;

(ii) For any $x, y, z \in G,(x y) z=x(y z)$;

(iii) There exists an element $e \in G$, called the identity, such that $x e=e x=x$ for any $x \in G$;

(iv) For any $x \in G$, there exists an inverse element $x^{-1} \in G$ such that $x x^{-1}=x^{-1} x=e$.

Preliminary examples of groups include $\mathbb{R}$ with the usual addiction, $\mathbb{C}^{*}$ with the usual multiplication and the groups $\omega_{n}$ of $n$-th roots of unity, also with multiplication as the binary operation. Some groups are continuous, while others are discrete; particularly in the former case, we might 
wish for some extra structure as a manifold, which brings us to the definition of a Lie group. At first glance, one might think that saying "a Lie group is a group with a differentiable manifold structure" suffices; however, we also need an additional condition.

Definition. A Lie group $G$ is a group with a differentiable manifold structure such that the operations $(x, y) \rightarrow x y$ and $x \mapsto x^{-1}$ are smooth throughout $G$. Its dimension is the dimension of the underlying manifold structure.

Remark 2.2.1. Sometimes, it is convenient to consider discrete groups as "zero-dimensional" manifolds with the discrete topology and talk about discrete Lie groups.

With this in mind, all our previous examples become Lie groups (the roots of unity being a discrete one). More interestingly, since the roots of unity are non-zero complex numbers, we have that $\omega_{n} \subset \mathbb{C}^{*}$; this gives rise to the notion of a subgroup, and of Lie subgroups.

Definition. Given a group $G$, a non-empty subset $H$ of $G$ is called a subgroup if it is a group in its own right with respect to the binary operation on $G$. If, furthermore, $G$ is a Lie group and $H$ is an immersed submanifold, we say that $H$ is a Lie subgroup.

Now, we briefly interrupt our definitions to explain what we are getting at. Our objective is to make $\mathcal{I}(\widehat{\mathbb{C}})$ a Lie group so that the symmetry group of a Julia set $J(R)$ is a subgroup of $\mathcal{I}(\widehat{\mathbb{C}})$, and then investigate what kind of subgroups are allowed. The next theorem - whose proof is far beyond the scope of this introduction - shall be crucial to help us along.

Theorem 2.2.1 (Closed Subgroup Theorem [HN12]). Let $G$ be a Lie group and $H \subset G$ a subgroup. If $H$ is closed, then it is an embedded Lie subgroup.

Another tool we shall need is to build adequate models of our symmetry groups - they might not be clearly tractable by themselves, so we must be able to borrow knowledge from more concrete examples. This leads us to the notion of a Lie isomorphism.

Definition. Let $G$ and $H$ be Lie groups. A function $\phi: G \rightarrow H$ is a Lie homomorphism if it is smooth, injective and preserves the group operation - i.e., $\phi(x y)=\phi(x) \phi(y)$ for every $x, y \in G$. If it is bijective with a smooth inverse, it is called a Lie isomorphism.

As an example, we consider two Lie groups. The first is the circle, $S^{1}=\{z \in \mathbb{C}:|z|=1\}$, with the group operation given by multiplication and the manifold structure given by its properties as a smooth curve in $\mathbb{C}$. Note that it is (quite trivially) a Lie group, since multiplication is analytic throughout $\mathbb{C}$, and its dimension is one. The second is the group of orientation-preserving orthogonal $2 \times 2$ matrices, $\mathrm{SO}(2)$, with the group operation given by matrix multiplication. It can be written as

$$
\mathrm{SO}(2)=\left\{\left(\begin{array}{cc}
\cos \theta & -\sin \theta \\
\sin \theta & \cos \theta
\end{array}\right): \theta \in[0,2 \pi)\right\},
$$

and so by writing $z=e^{i \theta}$ when $|z|=1$ we arrive at a very natural Lie group isomorphism given by

$$
e^{i \theta} \mapsto\left(\begin{array}{cc}
\cos \theta & -\sin \theta \\
\sin \theta & \cos \theta
\end{array}\right)
$$

As of now, we have precious little tools for effectively studying Lie groups. Thus, we now turn to a seemingly distinct topic - though the name "Lie" might offer some indication that it is, in fact, crucial to our discussion.

Definition. A Lie algebra is a vector space $V$ endowed with a Lie bracket $[\cdot, \cdot]: V \times V \rightarrow V$ satisfying:

(i) $(x, y) \mapsto[x, y]$ is a bilinear function;

(ii) $[x, y]=-[y, x]$ for $x, y \in V$; 
(iii) $[x,[y, z]]+[y,[z, x]]+[z,[x, y]]=0$.

Condition (ii) is known as the Jacobi identity; regarding notation, Lie algebras are usually denoted by lowercase fraktur letters - such as $\mathfrak{g}$. Our first example of a Lie algebra is $\mathbb{R}^{3}$ with the usual vector product $x \times y$. Our second example is a huge conceptual leap: we shall assign each Lie group a unique Lie algebra! First, of course, we shall need some tools.

Definition. Let $G$ be a Lie group and $\vec{X}$ a smooth vector field on $G$. It is said to be left-invariant if it is unchanged by left translations on the group. In other words, let $L_{g}(p)=g p$ be the left translation by $g$ on our Lie group. Then, $\vec{X}$ is left-invariant if $\vec{X} \circ L_{g}(p)=\left(d L_{g}\right)_{p} \vec{X}(p)$, where $\left(d L_{g}\right)_{p}$ denotes the differential of $L_{g}$ as a smooth self-map of $G$ calculated at $p$. The set of all left-invariant vector fields on $G$ is denoted $\mathfrak{X}^{L}(G)$.

The linearity of the differential operator implies that $\mathfrak{X}^{L}(G)$ is a vector space (it is, in fact, a linear subspace of $\mathfrak{X}(G)$ ), which means that we have a promising candidate for our Lie algebra. Now we need only find a Lie bracket.

Theorem 2.2.2. Let $\vec{X}$ and $\vec{Y}$ be left-invariant vector fields on $G$. Define $[\cdot, \cdot]: \mathfrak{X}^{L}(G) \times \mathfrak{X}^{L}(G) \rightarrow$ $\mathfrak{X}^{G}$ as

$$
[\vec{X}, \vec{Y}](p)=(d \vec{Y})_{p} \vec{X}(p)-(d \vec{X})_{p} \vec{Y}(p)
$$

by identifying vector fields with smooth $\mathbb{R}^{n}$-valued functions. Then, $\mathfrak{X}^{L}(G)$ endowed with $[\cdot, \cdot]$ is a Lie algebra.

Proof. It is clear that $[\vec{X}, \vec{Y}]=-[\vec{Y}, \vec{X}]$. To prove the Jacobi identity, we consider vector fields as derivations of real-valued functions:

$$
\vec{X} f(p)=\left.\frac{d}{d t}\right|_{t=0} f \circ \gamma(t)
$$

where $\gamma$ is a smooth curve on $G$ such that $\gamma(0)=p$ and $\gamma^{\prime}(0)=\vec{X}(p)$. With this, our bracket becomes

$$
[\vec{X}, \vec{Y}](f)=\vec{Y}(\vec{X} f)-\vec{X}(\vec{Y} f),
$$

and so (by suppressing the $f$ from our notation)

$[\vec{X},[\vec{Y}, \vec{Z}]]=[\vec{Y}, \vec{Z}] \vec{X}-\vec{X}[\vec{Y}, \vec{Z}]=(\vec{Z} \vec{Y}-\vec{Y} \vec{Z}) \vec{X}-\vec{X}(\vec{Z} \vec{Y}-\vec{Y} \vec{Z})=\vec{Z} \vec{Y} \vec{X}-\vec{Y} \vec{Z} \vec{X}-\vec{X} \vec{Z} \vec{Y}+\vec{X} \vec{Y} \vec{Z}$

Repeating this procedure for $[\vec{Y},[\vec{Z}, \vec{X}]]$ and $[\vec{Z},[\vec{X}, \vec{Y}]]$ and summing the three equations, we see the terms cancel out; thus $[\cdot, \cdot]$ satisfies the Jacobi identity. Lastly, we need to check that the bracket of two left-invariant fields is again left-invariant. We have:

$$
[\vec{X}, \vec{Y}] g=(\vec{Y} \vec{X}-\vec{X} \vec{Y}) g=\vec{Y} \vec{X} g-\vec{X} \vec{Y} g
$$

and since $\vec{X}$ and $\vec{Y}$ are left-invariant,

$$
[\vec{X}, \vec{Y}] g=\vec{Y}\left(d L_{g}\right) \vec{X}-\vec{X}\left(d L_{g}\right) \vec{Y} .
$$

Linearity of the differential then implies that $[\vec{X}, \vec{Y}] g=\left(d L_{g}\right)[\vec{X}, \vec{Y}]$, and we conclude that $[\cdot, \cdot]$ gives $\mathfrak{X}^{L}(G)$ a Lie algebra structure.

Remark 2.2.2. There is another way to construct the Lie algebra of a Lie group. Given $v \in T_{e} G$, the vector field $\vec{X}(p)=\left(d L_{p}\right)_{e} v$ is left-invariant; conversely, every left-invariant vector field can be expressed in this way for some $v \in T_{e} G$. Therefore, there exists an isomorphism between $T_{e} G$ and $\mathfrak{X}^{L}(G)$, and the bracket on $\mathfrak{X}^{L}(G)$ induces a bracket on $T_{e} G$ that promotes it to a Lie algebra.

Regarding notation, the Lie algebra of a Lie group is denoted by the same symbol as the group, in fraktur letters. As an example, we compute the Lie algebra $\mathfrak{s o}(3)$ of $\mathrm{SO}(3)$, the Lie group of $3 \times 3$ 
orientation-preserving orthogonal matrices. First, consider a smooth curve $A:(-\epsilon, \epsilon) \rightarrow \mathrm{SO}(3)$ such that $A(0)=I$. By the definition of $\mathrm{SO}(3)$, it satisfies $[A(t)]^{T} A(t)=I$ for all $t$, and we can differentiate this to obtain

$$
\left[A^{\prime}(t)\right]^{T} A(t)+[A(t)]^{T} A^{\prime}(t)=0 .
$$

In particular, this holds for $t=0$, and since $A(0)=I$ we conclude that $A^{\prime}(t) \in T_{e} \mathrm{SO}(3)$ is skewsymmetric. Therefore, $\mathfrak{s o}(3)$ is the set of $3 \times 3$ skew-symmetric matrices:

$$
\mathfrak{s o}(3)=\left\{\left(\begin{array}{ccc}
0 & x & y \\
-x & 0 & z \\
-y & -z & 0
\end{array}\right): x, y, z \in \mathbb{R}\right\}
$$

Although we won't prove it here, the Lie bracket is given by the usual matrix commutator $[A, B]=$ $B A-A B$. Proofs can be found in [HN12, Bak06].

As was our objective, the Lie algebra of a Lie group encodes valuable information about it. It allows us to pose geometric and topological questions on the Lie group in the language of linear algebra, starting with isomorphisms. We define a Lie algebra isomorphism $\psi: \mathfrak{g} \rightarrow \mathfrak{h}$ to be a linear isomorphism between vector spaces that preserves the bracket, i.e, $[\psi(v), \psi(w)]=\psi([v, w])$ for every $v, w \in \mathfrak{g}$.

Theorem 2.2.3. Let $G$ and $H$ be Lie groups related by a Lie group isomorphism $\phi: G \rightarrow H$. Then, $d \phi_{e}: \mathfrak{g} \rightarrow \mathfrak{h}$ is a Lie algebra isomorphism.

Proof. Take $X^{1}, X^{2} \in T_{e} G$ and extend them to left-invariant vector fields $\widetilde{X}^{1}, \widetilde{X}^{2}$ over $G$. Since $\phi$ is an isomorphism, we can push them forward under $\phi$ to obtain $\widetilde{Y}^{i}[\phi(g)]=(d \phi)_{g} \widetilde{X}^{i}(g)$, and these will be well-defined left-invariant vector fields. Then, $\left[\widetilde{Y}^{1}, \widetilde{Y}^{2}\right]=\widetilde{Y}^{2} \widetilde{Y}^{1}-\widetilde{Y}^{1} \widetilde{Y}^{2}=(d \phi) \widetilde{X}^{2}(d \phi) \widetilde{X}^{1}-$ $(d \phi) \widetilde{X}^{1}(d \phi) \widetilde{X}^{2}$. By the linearity of the differential of $\phi,\left[\widetilde{Y}^{1}, \widetilde{Y}^{2}\right]=(d \phi)\left[\widetilde{X}^{2}(d \phi) \widetilde{X}^{1}-\widetilde{X}^{1}(d \phi) \widetilde{X}^{2}\right]$. Finally, by the left-invariance of $\widetilde{X}^{i}$, we conclude that $\left[\widetilde{Y}^{1}, \widetilde{Y}^{2}\right]=(d \phi)\left(\widetilde{X}^{2} \widetilde{X}^{1}-\widetilde{X}^{1} \widetilde{X}^{2}\right)=(d \phi)\left[\widetilde{X}^{1}, \widetilde{X}^{2}\right]$, and we are done.

The converse is not, in general, true. For instance, $\mathfrak{s o}(3)$ is isomorphic as a Lie algebra to $\mathbb{R}^{3}$ with the vector product, but $\mathrm{SO}(3)$ is not isomorphic to $\mathbb{R}^{3}$. An important example of the theorem above is the case of a Lie subgroup. If $H \subset G$ is a Lie subgroup, the inclusion function $\imath: H \hookrightarrow H \subset G$ induces a Lie algebra isomorphism between $\mathfrak{h}$ and a Lie subalgebra of $\mathfrak{g}$. Therefore, the Lie algebra of a subgroup is a Lie subalgebra of the original group's Lie algebra.

Now, we consider Lie group homomorphisms $\gamma: \mathbb{R} \rightarrow G$. It is natural that $H=\gamma(\mathbb{R})$ is a Lie subgroup of $G$ of dimension one, which is why it is called a one-parameter subgroup of $G$. Furthermore, $d \gamma$ is a Lie algebra homomorphism between $\mathbb{R}$ and $\mathfrak{h} \subset \mathfrak{g}$ which, by the linearity of the differential, can be written as $d \gamma(t)=t X$ for some $X \in \mathfrak{h}$. We would like to relate these two morphisms; as it turns out, we can turn one into a "lift" of the other by means of the following theorem.

Theorem 2.2.4. Given any $X \in T_{e} G=\mathfrak{g}$, there exists a unique one-parameter subgroup $\gamma_{X}: \mathbb{R} \rightarrow$ $G$ such that $\gamma_{X}^{\prime}(0)=X$.

A proof of this result is quite technical, and involves integrating a distribution built upon the associated left-invariant vector field - interested readers can consult [AB15]. Leaving its proof aside, this theorem motivates the following definition.

Definition. The exponential map (sometimes called the Lie exponential) exp : $\mathfrak{g} \rightarrow G$ is defined as $\exp (X)=\gamma_{X}(1)$, where $\gamma_{X}$ is the one-parameter subgroup given by the theorem above.

Notice that $\gamma_{X}$ is, in fact, an integral curve of the left-invariant vector field generated by $X$. Indeed,

$$
\gamma_{X}^{\prime}(t)=\left.\frac{d}{d s} \gamma_{X}(t+s)\right|_{s=0}=d L_{\gamma_{X}(t)} X=X\left[\gamma_{X}(t)\right]
$$


Almost by construction, the exponential map provides us with a way to - as we promised - "lift" questions about one-parameter groups to questions about the Lie algebra of $G$.

Proposition 2.2.1. The exponential map satisfies the following properties for any $t$ in $\mathbb{R}$ and $X \in \mathfrak{g}$.

(i) $\exp (t X)=\gamma_{X}(t)$;

(ii) $\exp (-t X)=[\exp (t X)]^{-1}$;

(iii) $\exp \left(t_{1} X+t_{2} X\right)=\exp \left(t_{1} X\right) \exp \left(t_{2} X\right)$;

(iv) $\exp : T_{e} G \rightarrow G$ is smooth and $(d \exp )_{0}=\mathrm{id}$, and so exp is a diffeomorphism from an open neighbourhood of the origin in $T_{e} G$ onto a neighbourhood of the identity in $G$;

(v) Every connected one-parameter subgroup of $G$ can be parametrised as $\gamma(t)=\exp (t X)$ for some $X \in \mathfrak{g}$.

Proof. To prove (i), we must verify that $\gamma_{X}(t)=\gamma_{t X}(1)$. Consider $\gamma(s)=\gamma_{X}(s t)$, which is also a one-parameter subgroup. Differentiating at $s=0$ :

$$
\gamma^{\prime}(0)=t \gamma_{X}^{\prime}(s t)=t X,
$$

and the uniqueness of the one-parameter subgroup associated to a tangent vector implies that $\gamma_{X}(s t)=\gamma_{t X}(s)$. Choosing $s=1$ then yields (i). This also enables us to immediately prove (ii) and (iii) by recalling that $\gamma_{X}$ is a group homomorphism.

The proofs of (iv) and (v) are far more involved, and can be found in [AB15] or [HN12], so we provide only a quick sketch. In (iv), the idea is to construct a smooth vector field on $T G$, with flow $\varphi_{t}(g, X)=(g \exp (t X), X)$ and proving that it is smooth and complete. Then, $\exp (t X)$ becomes the projection of $\varphi_{t}(e, X)$ and hence is smooth. The fact that $\gamma_{X}$ is an integral curve of a flow implies that $(d \exp )_{0}$ is the identity. For $(\mathrm{v})$, let $\gamma$ be a one-parameter group with $\gamma^{\prime}(0)=X$. We take a convex, symmetric neighbourhood $U$ of $0 \in \mathfrak{g}$ such that $\left.\exp \right|_{U}$ is a diffeomorphism onto a neighbourhood of $e$. By continuity of $\gamma$, there exists an $\epsilon>0$ such that $\gamma([-\epsilon, \epsilon]) \subset \exp (U)$, and so we lift $\gamma$ to $\alpha(t)=\left[\left.\exp \right|_{U}\right]^{-1}[\gamma(t)]$ for $|t| \leq \epsilon$. What is left is to check that $\alpha(t)=t X$ for some $X \in \mathfrak{g}$, and then we apply property (iii) to show that $\gamma(t)=\exp (t X)$ for all $t$.

\subsection{Potential Theory}

The last tool we shall need concerns Green's functions and their generalisations; it is the study of subharmonic (or superharmonic) functions on $\mathbb{C}-$ and $\mathbb{C}^{n}$. We shall follow Ransford's approach [Ran95], with occasional input from Tsuji [Tsu75].

Of course, if all involved functions were $C^{2}$, we could simply say that a function $u: \Omega \subset \mathbb{C} \rightarrow \mathbb{R}$ is subharmonic if and only if $\nabla^{2} u=u_{x x}+u_{y y} \geq 0$, where $u_{x}$ denotes, as usual, partial differentiation with respect to $x$. Alas, this is not the case; we cannot even be sure a priori that the functions involved are continuous! Therefore, we shall define subharmonic functions through properties that do not depend on differentiability or continuity.

Definition. On a topological space $X$, a function $u: X \rightarrow[-\infty, \infty)$ is said to be upper semicontinuous if $\{x \in X: u(x)<a\}$ is open for every $a \in \mathbb{R}$. If $-u$ is upper semicontinuous, $u$ is said to be lower semicontinuous.

From the definition, every continuous function is both upper and lower semicontinuous. Upper semicontinuous functions also emulate some desirable properties of continuous functions:

Proposition 2.3.1. Let $u: X \rightarrow[-\infty, \infty)$ be an upper semicontinuous function. If $K \subset X$ is a compact subset, then $u$ is bounded above on $K$ and attains its bound. 
Proof. The sets $\{x \in X: u(x)<n\}_{n \geq 1}$ form an open cover of $X$, and so admit a finite subcover. This implies that $u$ is bounded above. Now, let $M=\sup _{K} u$. Then, the open sets $\{x \in X: u(x)<$ $M-1 / n\}_{n \geq 1}$ are not a cover of $X$ - they do not admit any finite subcover, by the definition of the supremum. Hence, there exists an $x \in X$ such that $u(x) \geq M-1 / n$ for every $n$, implying $u(x)=M$.

We are now ready to state the definition of a subharmonic function on the plane.

Definition. Let $\Omega$ be an open, non-empty subset of $\mathbb{C}$. A function $u: \Omega \rightarrow[-\infty, \infty)$ is called subharmonic if it is upper semicontinuous and satisfies the submean inequality: given $z \in \Omega$, there exists a $\rho>0$ such that

$$
u(z) \leq \frac{1}{2 \pi} \int_{0}^{2 \pi} u\left(z+r e^{i t}\right) d t
$$

for every $0 \leq r<\rho$. If $-u$ is subharmonic $u$ is said to be superharmonic.

It is clear from the definition that what usually passes as subharmonic functions - i.e., $C^{2}$ functions such that $\nabla^{2} u \geq 0$ - are subharmonic functions in this broader sense. Also, harmonic functions are subharmonic - in fact, a function is harmonic if and only if it is both sub- and superharmonic. Thus, many usual examples in the study of (sub)harmonic functions extend to this context.

Proposition 2.3.2. If $f: \Omega \rightarrow \mathbb{C}$ is holomorphic, then $u:=\log |f|$ is subharmonic.

Proof. Since $|f|$ and $\log$ are continuous functions, $u$ is upper semicontinuous. Also, if $z$ is not a root of $f, \log |f|$ is actually harmonic on $z$, and so satisfies the submean inequality. Lastly, if $f(z)=0$, then $u(z)=-\infty$ and the inequality is trivially satisfied.

This connection between harmonic and subharmonic functions is also further illustrated by the maximum principle.

Theorem 2.3.1 (The Maximum Principle). Let $u: \Omega \rightarrow[-\infty, \infty)$ be a subharmonic function on a domain $\Omega$ (here and throughout, a domain means a non-empty, open, connected subset of $\widehat{\mathbb{C}}$ ). If $u$ attains a global maximum on $\Omega$, then $u$ is constant. Furthermore, if $\limsup _{z \rightarrow w} u(z) \leq 0$ for all $w \in \partial \Omega$, then $u \leq 0$ on $\Omega$.

Proof. Suppose that $u$ attains a maximum $M$ on $\Omega$. We define the sets

$$
A=\{z \in \Omega: u(z)<M\} \quad \text { and } \quad B=\{z \in \Omega: u(z)=M\} .
$$

Since $u$ is upper semicontinuous by definition, $A$ is open. $B$, alas, is also open, for if $u(z)=M$ then, by the submean inequality, $u(w)=M$ for every $w$ in a small neighbourhood of $z$. Now, it is clear that $\Omega=A \cup B$, and $A \cap B=\emptyset$; since $\Omega$ is connected, we must have either $A=\Omega$ or $B=\Omega$. Since our assumption implies that $B$ is non-empty, we must have $B=\Omega$.

For the second part, extend $u$ to $\partial \Omega$ by setting $u(w)=\limsup _{z \rightarrow w} u(z)$ for any $w \in \partial \Omega$. Then $u$ is upper semicontinuous on $\bar{\Omega}$, which is compact (if necessary, we can consider $\Omega$ as a subset of $\widehat{\mathbb{C}}$ and let $\infty \in \partial \Omega$ ), and thus attains a maximum at some $w \in \bar{\Omega}$. If $w \in \partial \Omega$, then by assumption $u(w) \leq 0$, and so $u \leq 0$ in $\Omega$. If, on the other hand, $w \in \Omega$, then by the previous part of the theorem we know that $u$ is constant on $\Omega$, and so extends constantly to $\bar{\Omega}$. By assumption, it follows that $u \leq 0$ on $\Omega$.

We end this comparison with the usual subharmonic function by showing that smooth subharmonic functions are indeed the ones we are thinking of.

Proposition 2.3.3. Suppose $u: \Omega \rightarrow[-\infty, \infty)$ is of class $C^{2}$. Then, $u$ is subharmonic if and only if $\nabla^{2} u \geq 0$. 
Proof. One side follows immediately from the classical submean inequality for functions satisfying $\nabla^{2} u \geq 0$. For the other, suppose by contradiction that there exists some $z \in \Omega$ such that $\nabla^{2} u(z)<0$. By continuity, there is some $\rho>0$ such that $\nabla^{2} u(w) \leq 0$ for $|z-w|<\rho$, and so $u$ is actually superharmonic on $B(z ; \rho)$ - and hence harmonic there. In particular, $\nabla^{2} u(z)=0$, contradicting our original assumption.

Next, we prove two theorems on how to obtain new subharmonic functions from old ones.

Theorem 2.3.2. Let $(\Omega, \mu)$ be a measure space such that $\mu(\Omega)<\infty$. Let $U$ be an open subset of $\mathbb{C}$, and $v: U \times \Omega \rightarrow[-\infty, \infty)$ a measurable function satisfying:

(i) $z \mapsto v(z, w)$ is subharmonic on $U$ for every $w \in \Omega$;

(ii) $z \mapsto \sup w \in \Omega v(z, w)$ is locally bounded above on $U$.

Then $u(z):=\int_{\Omega} v(z, w) d \mu(w)$ is subharmonic on $U$.

Proof. Since subharmonicity is a local property, it suffices to show it on every relatively compact subdomain $D$ of $U$. Let $D$ be such a subdomain. Then, property (ii) implies that $\sup _{w \in \Omega} v(z, w)$ is bounded above on $D$, and so - subtracting a constant if necessary - we can assume that $v \leq 0$ on $D \times \Omega$. We are, therefore, free to use Fatou's lemma and Fubini's theorem. Taking a sequence $z_{n}$ in $D$ such that $z_{n} \rightarrow z$, we get by Fatou's lemma that

$$
\limsup _{n \rightarrow \infty} u\left(z_{n}\right) \leq \int_{\Omega} \limsup _{n \rightarrow \infty} v\left(z_{n}, w\right) d \mu(w) .
$$

Since $v$ is upper semicontinuous on the first argument, it follows that

$$
\limsup _{n \rightarrow \infty} u\left(z_{n}\right) \leq \int_{\Omega} v(z, w) d \mu(w)=u(z),
$$

and thus $u$ is upper semicontinuous. Now, if $\bar{B}(z, \rho) \subset D$, we have by Fubini's theorem:

$$
\frac{1}{2 \pi} \int_{0}^{2 \pi} u\left(z+\rho e^{i \theta}\right) d \theta=\int_{\Omega}\left[\int_{0}^{2 \pi} v\left(z+\rho e^{i \theta}, w\right) d \theta\right] d \mu(w) .
$$

Since $v$ is subharmonic on the first argument, we have that the inner integral is bonded below by $v(z, w)$ for every $w \in \Omega$, and since $\mu(\Omega)$ is finite we conclude that $u$ satisfies the submean inequality.

Theorem 2.3.3. Let $f: \Omega_{1} \rightarrow \Omega_{2}$ be a holomorphic function with $\Omega_{1}, \Omega_{2}$ open subsets of $\mathbb{C}$. If $u$ is subharmonic on $\Omega_{2}$, then $f^{*} u=u \circ f$ is subharmonic on $\Omega_{1}$.

Proof. Again, it is enough to show the result for a relatively compact subdomain $D_{1} \subset \Omega_{1}$. Set $D_{2}=f\left(D_{1}\right)$. We shall now employ a result we have not proved: for every subharmonic function on a domain, there exists a sequence $\left(u_{n}\right)_{n \geq 1}$ of smooth subharmonic functions that approaches it monotonically from above (a proof can be found in [Ran95]; it involves convoluting $u$ with a sequence of smoothing kernels). Hence, we take a sequence $\left(u_{n}\right)_{n \geq 1} \subset C^{\infty}\left(D_{2}\right)$ of subharmonic functions such that $u_{n} \searrow u$ on $D_{2}$. Since they are smooth, we know that they must satisfy $\nabla^{2} u_{n} \geq 0$. By the chain rule,

$$
\nabla^{2}\left(u_{n} \circ f\right)=\left[\left(\nabla^{2} u_{n}\right) \circ f\right]\left|f^{\prime}\right|^{2}
$$

on $D_{1}$, and hence $\nabla^{2}\left(u_{n} \circ f\right) \geq 0$ and $u_{n} \circ f$ is subharmonic on $D_{1}$ for all $n$. Letting $n \rightarrow \infty$, we see that $u \circ f$ is subharmonic on $D_{1}$ - the monotone convergence theorem implies that $u \circ f$ satisfies the submean inequality, and it is upper semicontinuous because the set $\left\{z \in D_{1}: u \circ f(z)<\alpha\right\}$ is the countable union of the open sets $\left\{z \in D_{1}: u_{n} \circ f(z)<\alpha\right\}$ for all $n$.

Now, we are ready to actually define potentials. 
Definition. Let $\mu$ be a finite Borel measure on $\mathbb{C}$ with compact support. Its potential is the function $p_{\mu}: \mathbb{C} \rightarrow[-\infty, \infty)$ defined as

$$
p_{\mu}(z)=\int_{\mathbb{C}} \log |z-w| d \mu(w) .
$$

The term inside the integral is, as we know, subharmonic. In fact, we can draw upon our previous considerations to prove more.

Proposition 2.3.4. $p_{\mu}$ is subharmonic throughout $\mathbb{C}$, harmonic on $\mathbb{C} \backslash(\operatorname{supp} \mu)$ and satisfies

$$
p_{\mu}(z)=\mu(\mathbb{C}) \log |z|+O\left(|z|^{-1}\right)
$$

as $z \rightarrow \infty$

Proof. Set $K=\operatorname{supp} \mu$. We can regard $\mu$ as a measure on $K$, and so we can consider the function $v(z, w)=\log |z-w|$ on $\mathbb{C} \times K$. Since $v$ is subharmonic on $z$ and $\mu$ is a finite measure, we conclude that $p_{\mu}$ is subharmonic on $\mathbb{C}$. Applying the same rationale to $v(z, w)=-\log |z-w|$, we can conclude that $p_{\mu}$ is superharmonic on $(\mathbb{C} \backslash K) \times K$, and therefore harmonic there.

Now, write $\log |z-w|=\log (|z||1-w / z|)=\log |z|+\log |1-w / z|$ for $z \neq 0$. By linearity, the integral becomes:

$$
p_{\mu}(z)=\int_{\mathbb{C}} \log |z| d \mu(w)+\int_{\mathbb{C}} \log |1-w / z| d \mu(w) .
$$

Since the first term does not depend on $z$, it reduces to $\mu(\mathbb{C}) \log |z|$; as for the second, the fact that $\mu$ is supported on $K$, which is a bounded set, implies that it behaves as $O\left(|z|^{-1}\right)$ as $|z|$ grows to infinity.

By definition, we have allowed $p_{\mu}$ to take on minus infinity as a value. However, we can show that if $p_{\mu}$ is strictly finite on the support of $\mu$, it is so throughout all of $\mathbb{C}$. More specifically, we have the following.

Theorem 2.3.4 (Minimum Principle). Let $\mu$ be a finite Borel measure on $\mathbb{C}$ with compact support $K$. If $p_{\mu} \geq M$ on $K$ for some $M \in \mathbb{R}$, then $p_{\mu} \geq M$ on all of $\mathbb{C}$.

Proof. Let $u=-p_{\mu}$ on $\mathbb{C} \backslash K$. Then, $u$ is harmonic and goes to minus infinity as $z \rightarrow \infty$. Now, set $\Omega_{n}=\{z \in \mathbb{C} \backslash K: u(z)>-n\}$. Since $u$ is harmonic, and hence smooth, on $\mathbb{C} \backslash K$ and $K$ is bounded it follows that for large enough $n$ the set $\Omega_{n} \cup K$ is a simply connected subset of $\mathbb{C}$. In other words, the boundary of $\Omega_{n}$ consists of $\partial K$ and a large topological circle. Also if $n$ is large enough, $\lim \sup _{z \rightarrow \zeta} u(z) \leq \limsup _{z \rightarrow w} u(z)$ for any $\zeta$ and $w$ in the outer boundary of $\Omega_{n}$ and $\partial K$, respectively. Hence, we conclude that $\limsup _{z \rightarrow w} u(z) \leq-M$ for any $w \in \partial \Omega_{n}$, and by the maximum principle it follows that $u \leq-M$ and $p_{\mu} \geq M$ on $\Omega_{n}$. By letting $n \rightarrow \infty$, we conclude that $p_{\mu} \geq M$ on $\mathbb{C} \backslash K$, and we are done.

A powerful physical motivation for the study of potentials is the question of equidistributing electrical charges throughout a rigid body. In this setting, the support $K$ of our measure $\mu$ represents the body, and $\mu$ itself is the charge distribution. This makes our potential $p_{\mu}$ into the "electric" potential associated to the distribution of charges; as with a bona fide electric field, we expect the integral of the potential to represent the total energy of the system.

Definition. Let $\mu$ be a finite Borel measure with compact support $K \subset \mathbb{C}$. Its energy is defined as

$$
I(\mu)=\int_{\mathbb{C}} p_{\mu}(z) d \mu(z)=\int_{\mathbb{C}} \int_{\mathbb{C}} \log |z-w| d \mu(w) d \mu(z) .
$$

From the definition, it is possible that $I(\mu)=-\infty$. Indeed, for certain compact sets $K$ this is the best one can possibly hope for. These sets deserve a name of their own. 
Definition. A set $E \subset \mathbb{C}$ is called polar if $(\mu)=-\infty$ for every finite Borel measure whose support is a compact subset of $E$. For a subset $S$ of $\mathbb{C}$, a property is said to hold nearly everywhere (n.e.) on $S$ if it holds on $S \backslash E$ and $E$ is a Borel polar set.

Clearly, every subset of a polar set is polar; also, singletons are our first concrete example of a polar set. Now, we show that polar sets are, in some sense, small.

Theorem 2.3.5. Let $\mu$ be a finite Borel measure such that $I(\mu)>-\infty$. Then, every Borel polar set $E$ satisfies $\mu(E)=0$.

Proof. Suppose that there exists a Borel polar set $E$ with $\mu(E)>0$. We shall show that $E$ is not polar. Since $\mu$ is regular, we can take a compact subset $K$ of $E$ such that $\mu(K)>0$ as well. Set $\tilde{\mu}=\left.\mu\right|_{K}$ and $d=\operatorname{diam}(\operatorname{supp} \mu)$. Now, $\tilde{\mu}$ is a finite non-zero measure whose support is a compact subset of $E$, and it satisfies

$$
I(\tilde{\mu})=\int_{K} \int_{K} \log \left|\frac{z-w}{d}\right| d \mu(z) d \mu(w)+\mu(K)^{2} \log d .
$$

Since $|(z-w) / d| \leq 1$ for any $z, w$ where $\mu$ has non-zero density, we can write

$$
I(\tilde{\mu}) \geq \int_{\mathbb{C}} \int_{\mathbb{C}} \log \left|\frac{z-w}{d}\right| d \mu(z) d \mu(w)+\mu(K)^{2} \log d,
$$

which reduces to $I(\tilde{\mu})=I(\mu)-\mu(\mathbb{C})^{2} \log d+\mu(K)^{2} \log d>-\infty$ and we conclude that $E$ is nonpolar.

This result sheds some light as to the nature of polar sets. We see first that they can be called small in a true measure-theoretic sense, and second that cardinality-wise "small" sets are polar.

Corollary. Every Borel polar set has Lebesgue measure zero.

Proof. We want to show that, for every $\rho>0$, the measure $d \mu=\left.d A\right|_{B(0 ; \rho)}$ has finite energy, where $A$ denotes the Lebesgue measure of the plane. Then, the above theorem implies that any Borel polar set $E$ satisfies $\mu(E)=0$, and so $E \cap B(0 ; \rho)$ has Lebesgue measure zero. Letting $\rho \rightarrow 0$ will then give us our final result.

Thus, let $\rho>0$ and define $d \mu=\left.d A\right|_{B(0 ; \rho)}$. We have

$$
p_{\mu}(z)=\int_{B(0 ; \rho)} \log |z-w| d A(w),
$$

and using the same trick as in the previous theorem we get

$$
p_{\mu}(z)=\int_{B(0 ; \rho)} \log \left|\frac{z-w}{2 \rho}\right| d A(w)+\pi \rho^{2} \log (2 \rho) .
$$

Now, since the term inside the integral is again negative for any relevant values of $z$ and $w$, we get

$$
p_{\mu}(z) \geq \int_{0}^{2 \pi} \int_{0}^{2 \rho} \log \left(\frac{r}{2 \rho}\right) r d r d t+\pi \rho^{2} \log (2 \rho),
$$

and we evaluate it to obtain $p_{\mu}(z) \geq-2 \pi \rho^{2}+\pi \rho^{2} \log (2 \rho)$. Thus,

$$
I(\mu)=\int_{B(0 ; \rho)} p_{\mu}(z) d z \geq \pi \rho^{2}\left[-2 \pi \rho^{2}+\pi \rho^{2} \log (2 \rho)\right]>-\infty
$$

and we are done.

Corollary. A countable union of Borel polar sets is polar. In particular, any countable subset of $\mathbb{C}$ is polar. 
Proof. Suppose that $E_{n}$ are Borel polar sets for every $n \geq 1$ and take $E=\cup_{n} E_{n}$. Let $\mu$ be a finite Borel measure whose support is a compact subset of $E$. If $\mu$ has finite energy, then $\mu\left(E_{n}\right)=0$ for all $n$. By the additivity of the measure, this implies that $\mu(E)=0$, and thus $\mu$ is the zero measure. It follows that $E$ is polar.

Now, carrying on with our physical analogy of the potential, we want to obtain measures that maximise the system's energy - since our definition of energy is missing a minus sign, we are not maximising it as would be customary for physicists. We have:

Definition. Let $K$ be a compact subset of $\mathbb{C}$. An equilibrium measure for $K$ is a measure $\mu$ supported on $K$ such that

$$
I(\mu)=\sup _{\nu \in \mathcal{P}(K)} I(\nu),
$$

where $\mathcal{P}(K)$ denotes - here and throughout the text - the set of all probability measures supported on $K$.

In order to study existence and uniqueness of an equilibrium measure, we endow the space $\mathcal{P}(K)$ with the weak - or weak ${ }^{*}$ - topology. This is the topology obtained by saying that $\mu_{n} \rightarrow \mu$ if, and only if,

$$
\int_{K} f d \mu_{n} \rightarrow \int_{K} f d \mu
$$

for every continuous, real-valued function on $K$. It is a non-trivial exercise - we refer to [vG] for details- to prove that, since $K$ is compact, it follows that $\mathcal{P}(K)$ is compact in the weak topology. Hence, the existence of at least one equilibrium measure for every compact $K \subset \mathbb{C}$ follows from the next result.

Proposition 2.3.5. If $\mu_{n} \rightarrow \mu$ in $\mathcal{P}(K)$, then $\limsup _{n \rightarrow \infty} I\left(\mu_{n}\right) \leq I(\mu)-$ or, equivalently, $I$ is upper semicontinuous in the weak topology.

Proof. Let $\phi, \psi$ be continuous functions on $K$. By the definition of weak convergence, and using Fubini's theorem, it follows that

$$
\int_{K} \int_{K} \phi(z) \psi(w) d \mu_{n}(z) d \mu_{n}(w) \rightarrow \int_{K} \int_{K} \phi(z) \psi(w) d \mu(z) d \mu(w) .
$$

Now, using the Stone-Weiestrass theorem, we see that every continuous function $\chi$ on $K \times K$ can be uniformly approximated by finite sums of the form

$$
\sum_{j} \phi_{j}(z) \psi_{j}(w)
$$

where the $\phi_{j}$ and $\psi_{j}$ are continuous functions on $K$. Therefore, for every continuous function $\chi(z, w)$, we have

$$
\int_{K} \int_{K} \chi(z, w) d \mu_{n}(z) d \mu_{n}(w) \rightarrow \int_{K} \int_{K} \chi(z, w) d \mu(z) d \mu(w) .
$$

It would be great if we could apply this to the function $\log |z-w|$. However, this function is not continuous on $K \times K$ - it is merely upper semicontinuous. Thus, we shall approximate it by a monotone sequence of continuous functions. Pick $\chi_{m}(z, w)=\max (\log |z-w|,-m)$. Then, $\chi_{1} \geq \chi_{2} \geq \cdots \geq \chi$, and $\chi_{m} \rightarrow \chi$ as $m \rightarrow \infty$. We can write

$$
\int_{K} \int_{K} \chi(z, w) d \mu_{n}(z) d \mu_{n}(w) \leq \int_{K} \int_{K} \chi_{m}(z, w) d \mu_{n}(z) d \mu_{n}(w),
$$

and so

$$
\limsup _{n \rightarrow \infty} I\left(\mu_{n}\right) \leq \limsup _{n \rightarrow \infty} \int_{K} \int_{K} \chi_{m}(z, w) d \mu_{n}(z) d \mu_{n}(w) .
$$


Since the $\chi_{m}$ are continuous and $\mu_{n} \rightarrow \mu$ weakly, the inequality becomes

$$
\limsup _{n \rightarrow \infty} I\left(\mu_{n}\right) \leq \int_{K} \int_{K} \chi_{m}(z, w) d \mu(z) d \mu(w)
$$

and finally the term on the right converges to $I(\mu)$ as $m \rightarrow \infty$ by the monotone convergence theorem.

As we know, an upper semicontinuous function on a compact set is bounded above and attains its maximum. Thus, we conclude that any compact $K \subset \mathbb{C}$ has at least one equilibrium measure. Next, we show how the potentials associated to such measures can be expected to behave.

Theorem 2.3.6 (Frostman). Let $K$ be a compact subset of $\mathbb{C}$, and $\mu$ an equilibrium measure for K. Then,

(i) $p_{\mu} \geq I(\mu)$ on $\mathbb{C}$;

(ii) $p_{\mu}=I(\mu)$ nearly everywhere on $K$.

Proof. This is one of the central theorems in potential theory, and its proof is quite lengthy. As such, we will provide only an idea of the proof; details can be found in [Ran95].

If $I(\mu)=-\infty$ - i.e., if $K$ is polar -, then the result is trivially true. Thus, we assume that $I(\mu)>-\infty$. Now, we show how the result follows from two similar assertions:

(a) $K_{n}:=\left\{z \in K: p_{\mu}(z) \geq I(\mu)+1 / n\right\}$ is polar for every $n \geq 1$;

(b) $L_{n}:=\left\{z \in \operatorname{supp} \mu: p_{\mu}(z)<I(\mu)-1 / n\right\}$ is empty for every $n \geq 1$.

First, (b) implies that $p_{\mu} \geq I(\mu)$ on supp $\mu$, and so the maximum principle gives us (i). Next, since $p_{\mu}$ is upper semicontinuous, every $K_{n}$ is closed, and so $E=\cup_{n} K_{n}$ is an $F_{\sigma}$ set. If (a) holds, then $E$ - as a countable collection of polar sets - is polar, and combining the definition of the $K_{n}$ 's with (i) we get that $p_{\mu}=I(\mu)$ on $K \backslash E$.

Our next order of business is to prove the uniqueness of the equilibrium measure for non-polar sets - on a polar set, every measure is an equilibrium one, for they all satisfy $I(\mu)=-\infty$. In order to do that, however, we shall have to differentiate potentials - which is a problem, since they may not differentiable in the usual sense. Thus, we must regard potentials as distributions.

Definition. A distribution is a continuous linear functional defined on the space of $C^{\infty}$ real-valued functions with compact support (also called the space of test functions).

Since old-school differentiation of potentials is out of the question, we turn to integration. In order for this to make sense, we must reassure ourselves that potentials can be integrated without blowing up to infinity. In fact, we go all the way and prove it for general subharmonic functions.

Theorem 2.3.7. Let $u$ be a subharmonic function on a domain $\Omega \subset \mathbb{C}$, and assume that $u$ is not constantly $-\infty$. Then $u$ is locally integrable, i.e., $\int_{K}|u| d A<\infty$ for every compact subset $K$ of $\Omega$.

Proof. Since $K$ is compact, it suffices to show the theorem for small balls centred on any $w \in \Omega$. In other words, we must show that for every $w \in \omega$ there exists a $\rho>0$ such that

$$
\int_{B(w ; \rho)}|u| d A<\infty
$$

Let $A$ denote the set of all $w \in \Omega$ possessing this property, and $B$ the set of $w \in \Omega$ not satisfying it. Clearly, $A$ and $B$ partition $\Omega$; then, by proving that they are both open, the connectedness of $\Omega$ implies that one of them is empty. We prove that $u \equiv-\infty$ on $B$, and the proof shall be done. 
Let $w \in A$, and choose $\rho>0$ such that $\int_{B(w ; \rho)}|u| d A$ is finite. Given $w^{\prime} \in B(w ; \rho)$, set $\rho^{\prime}=$ $\rho-\left|w-w^{\prime}\right|$. Then, $B\left(w^{\prime} ; \rho^{\prime}\right) \subset B(w ; \rho)$, meaning that

$$
\int_{B\left(w^{\prime}, \rho^{\prime}\right)}|u| d A<\infty
$$

and so $B(w ; \rho) \subset A$ and $A$ is open.

Now, let $w \in B$, and choose $\rho$ such that $\bar{B}(w, 2 \rho) \subset \Omega$. As $w \in B$, we have that

$$
\int_{B(w ; \rho)}|u|=\infty
$$

Take $w^{\prime} \in B(w ; \rho)$ and set $w^{\prime}=\rho+\left|w-w^{\prime}\right|$. Then, $B(w, \rho) \subset B\left(w^{\prime} ; \rho^{\prime}\right)$ and, as $u$ is bounded above on $\bar{B}\left(w^{\prime} ; \rho^{\prime}\right)$, we conclude that

$$
\int_{B\left(w^{\prime} ; \rho^{\prime}\right)} u d A=-\infty
$$

Now, $u$ satisfies the submean inequality; hence,

$$
u\left(w^{\prime}\right) \leq \frac{1}{2 \pi} \int_{0}^{2 \pi} u\left(w^{\prime}+r e^{i t}\right) d t
$$

for $0 \leq r<\rho^{\prime}$. Multiplying the inequality by $2 \pi r$ and integrating from $r=0$ to $r=2 \pi$, we obtain

$$
\pi \rho^{\prime 2} u\left(w^{\prime}\right) \leq \int_{B\left(w^{\prime} ; \rho^{\prime}\right)} u d A=-\infty .
$$

Thus, $u \equiv-\infty$ on $B(w, \rho)$. This shows both that $B(w, \rho) \subset B-$ and so $B$ is open - and that $u \equiv-\infty$ on $B$, which means that $B$ is empty and we are done.

Now, if we are given a subharmonic function $u$ on a domain $\Omega$, we can promote it to a distribution since for every test function $\varphi: \mathbb{C} \rightarrow \mathbb{R}$ the integral

$$
\int_{\Omega} u \varphi d A
$$

is finite. This means that subharmonic functions can be derived in the sense of distributions, and this is how we are going to differentiate them from now on.

Definition. Let $u$ be a subharmonic function on a domain $\Omega$. Denote by $C_{c}^{\infty}(\Omega)$ the space of all real-valued smooth functions whose support is a compact subset of $\Omega$. The generalised Laplacian of $u$ is the distribution $\nabla^{2} u$ such that

$$
\int_{\Omega} \nabla^{2} u \cdot \varphi d A=\int_{\Omega} u \cdot \nabla^{2} \varphi d A \text { for every } \varphi \in C_{c}^{\infty}(\Omega) .
$$

Of course, even though the right-hand side of the equation above makes sense, this does not mean that the distribution $\nabla^{2} u$ exists. Our first order of business, then, should be to prove the existence and uniqueness of the generalised Laplacian; however, the proof is long and involved, and so we will not show it here. Suffice to say that it relies heavily on the Riesz representation theorem and the fact that any continuous function can be approximated by smooth ones. As a by-product of the proof, we get that $\nabla^{2} u$ is a positive linear functional - i.e.,

$$
\int_{\Omega} \nabla^{2} u \cdot \varphi d A \geq 0
$$

whenever $\varphi$ is non-negative throughout $\Omega$. In other words, we get that $\nabla^{2} u \geq 0$ - a "straightforward" generalisation of the condition for $C^{2}$ subharmonic functions. This means that $\nabla^{2} u$ can actually be 
regarded as a bona fide measure.

Proposition 2.3.6. If $T$ is a distribution on $\Omega$ and $T(\varphi) \geq 0$ for any non-negative test function $\varphi$, then there exists a finite Borel measure $\mu$ on $\Omega$ such that

$$
T(\varphi)=\int_{\Omega} \varphi d \mu
$$

Proof. The Riesz representation theorem ensures the result for linear functionals defined on $C^{0}(\Omega)$. Thus, we need only extend $T$ to all continuous functions instead of just smooth ones. Since $C_{c}^{\infty}(\Omega)$ is dense in $C_{c}^{0}(\Omega)$ (something we shall not prove), given $\varphi \in C_{c}^{0}(\Omega)$ we can take a sequence $\varphi_{n} \in C_{c}^{\infty}(\Omega)$ such that $\varphi_{n} \rightarrow \varphi$, and define

$$
T(\varphi)=\lim _{n \rightarrow \infty} T\left(\varphi_{n}\right) .
$$

In order to ensure that $T(\varphi)$ is well-defined, we must prove that the limit exists and is independent of the approximating sequence. Let $\varphi_{n}$ and $\tilde{\varphi}_{n}$ be two such sequences, and pick a compact subset $K \subset \Omega$ such that supp $\varphi_{n}$ and $\operatorname{supp} \tilde{\varphi}_{n}$ are contained in $K$ for all $n$. Now, choose $\psi \in C_{c}^{\infty}(\Omega)$ such that $0 \leq \psi \leq 1$. Now, for all $m, n \geq 1$, we have

$$
\left|\varphi_{m}-\tilde{\varphi}_{n}\right| \leq\left\|\varphi_{m}-\tilde{\varphi}_{n}\right\|_{K} \psi
$$

throughout $\Omega$, where $\left\|\varphi_{m}-\tilde{\varphi}_{n}\right\|_{K}:=\sup _{z \in K}\left|\varphi_{m}-\tilde{\varphi}_{n}\right|$. Now, since $T$ is a positive distribution, we get

$$
\left|T\left(\varphi_{m}\right)-T\left(\tilde{\varphi}_{n}\right)\right| \leq\left\|\varphi_{m}-\tilde{\varphi}_{n}\right\|_{K} T(\psi) .
$$

Since both $\varphi_{m}$ and $\tilde{\varphi}_{n}$ tend to $\phi$ as $m, n \rightarrow \infty$, the right side of this inequality goes to zero, and so the sought-after limit exists - just consider the inequality above with $\varphi_{n}=\tilde{\varphi}_{n}$. Finally, it is also independent of the choice of approximating sequence - consider the inequality with $m=n$.

Throughout the text, we identify a positive distribution with its corresponding finite Borel measure. Thus, we can say that if $u$ is subharmonic then $\nabla^{2} u$ is a measure, or even write equalities involving distributions and measures. Our first such example is the theorem below.

Theorem 2.3.8. For a finite Borel measure $\mu$ on $\mathbb{C}$ with compact support, $\nabla^{2} p_{\mu}=2 \pi \mu$.

Proof. Given $\varphi \in C_{c}^{\infty}(\mathbb{C})$, we have

$$
\int_{\mathbb{C}} p_{\mu} \nabla^{2} \varphi d A=\int_{\mathbb{C}}\left[\int_{\mathbb{C}} \log |z-w| d \mu(w)\right] \nabla^{2} \varphi(z) d A(z) .
$$

Since $\nabla^{2} \varphi$ is bounded and $\log |z|$ is locally integrable with respect to Lebesgue, we can use Fubini's theorem to obtain

$$
\int_{\mathbb{C}} p_{\mu} \nabla^{2} \varphi d A=\int_{\mathbb{C}}\left[\int_{\mathbb{C}} \log |z-w| \nabla^{2} \varphi(z) d A\right] d \mu(w) .
$$

Now, we take a closer look at the inner integral. Since it is an improper integral, we write

$$
\int_{\mathbb{C}} \log |z-w| \nabla^{2} \varphi(z) d A(z)=\lim _{\epsilon \rightarrow 0} \int_{|z-w|>\epsilon} \log |z-w| \nabla^{2} \varphi(z) d A(z)
$$

and by Green's theorem this becomes

$$
\left.\lim _{\epsilon \rightarrow 0} \int_{0}^{2 \pi}\left[\varphi\left(w+r e^{i t}\right)-r \log r \frac{\partial \varphi}{\partial r}\left(w+r e^{i t}\right)\right]\right|_{r=\epsilon} d t
$$

As $\epsilon \rightarrow 0$, this becomes $2 \pi \varphi(w)$, thus

$$
\int_{\mathbb{C}} p_{\mu} \nabla^{2} \varphi d A=\int_{\mathbb{C}} 2 \pi \varphi d \mu
$$


and the conclusion follows by the uniqueness of the generalised Laplacian.

An immediate corollary of this theorem shall be the cornerstone of our proof of uniqueness for equilibrium measures, and it is also the reason why we took this detour.

Corollary. Let $\mu_{1}$ and $\mu_{2}$ be finite Borel measures on $\mathbb{C}$ with compact support. If $p_{\mu_{1}}=p_{\mu_{2}}+h$ on any open set $U$, where $h$ is harmonic on $U$, then $\left.\mu_{1}\right|_{U}=\left.\mu_{2}\right|_{U}$.

Proof. Since $h$ is harmonic, we differentiate the equality (in the sense of distributions) to obtain

$$
\left.\left(\nabla^{2} p_{\mu_{1}}\right)\right|_{U}=\left.\left(\nabla^{2} p_{\mu_{2}}\right)\right|_{U}
$$

The conclusion follows from the previous theorem.

Finally, our much-awaited proof of uniqueness.

Theorem 2.3.9. Let $K$ be a compact non-polar subset of $\mathbb{C}$. Then, $K$ admits an unique equilibrium measure $\mu$.

Proof. Since $K$ is compact, we already know that equilibrium measures exist. Thus, suppose $\mu$ and $\nu$ are two distinct equilibrium measures. By Frostman's theorem, $p_{\mu} \geq I(\mu)$, and equality holds nearly everywhere on $K$. Since $\nu$ is also an equilibrium measure, we can apply the same reasoning, and since $I(\mu)=I(\nu)$ we conclude that $p_{\mu}=p_{\nu}$ nearly everywhere on $K$.

Next, we look at the bounded components of $\mathbb{C} \backslash K$. Since $p_{\mu}$ and $p_{\nu}$ are harmonic there, the maximum principle implies that $p_{\mu}=p_{\nu}=I(\mu)$ on every such component. Finally, for the unbounded component of $\mathbb{C} \backslash K$, notice that the difference $p_{\mu}-p_{\nu}$ is harmonic and bounded, and so by Liouville's theorem it is constant and equal to $I(\mu)-I(\nu)=0$.

The existence of equilibrium measures will serve as the basis for the existence of one of our most powerful tools: the Green's function.

Definition. Let $\Omega$ be a domain in $\widehat{\mathbb{C}}$. A Green's function for $\Omega$ is a function $g_{\Omega}: \Omega \times \Omega \rightarrow(-\infty, \infty]$ such that:

(i) The function $z \rightarrow g_{\Omega}(\cdot, w)$ is harmonic on $\Omega \backslash\{w\}$;

(ii) For n.e. $\zeta \in \partial \Omega, g_{\Omega}(z, w) \rightarrow 0$ as $z \rightarrow \zeta$;

(iii) $g_{\Omega}(w, w)=\infty$, and

$$
g_{\Omega}(z, w)=\left\{\begin{array}{ll}
-\log |z-w|+O(1), & w \neq \infty \\
\log |z|+O(1), & w=\infty
\end{array} .\right.
$$

We justify the above definition - and most of the work done here on equilibrium measures through the following theorem.

Theorem 2.3.10. Let $\Omega$ be a domain in $\widehat{\mathbb{C}}$ with non-polar boundary. Then, there exists a unique Green's function for $\Omega$.

Proof. First, we prove uniqueness. If $g_{1}$ and $g_{2}$ are two Green's functions for $\Omega$, then $h(z)=$ $g_{1}(z, w)-g_{2}(z, w)$ is, for any fixed $w$, harmonic and bounded on $\Omega \backslash\{w\}$. Also, $h$ goes to zero at nearly every point of the boundary, and so by the maximum principle $h \equiv 0$.

Next, we prove existence for $w=\infty$ (so $\Omega$ is unbounded). Let $K=\widehat{\mathbb{C}} \backslash \Omega$; immediately, we see that $K$ is a compact subset of $\mathbb{C}$, and its boundary is non-polar. Therefore, there exists an unique equilibrium measure $\mu$ supported on $K$. Its potential, $p_{\mu}$, is harmonic throughout $\widehat{\mathbb{C}} \backslash K=\Omega$, and it also goes towards infinity as

$$
p_{\mu}(z)=\log |z|+O\left(|z|^{-1}\right) .
$$


All we are missing is that it tend to zero as $z$ approaches the boundary. By Frostman's theorem, however, we know that $p_{\mu}(z)=I(\mu)$ nearly everywhere on $K$, and particularly on $\partial K=\partial \Omega$. Therefore, $g_{\Omega}(z, w)=p_{\mu}(z)-I(\mu)$ is the Green's function for $\Omega$.

Finally, the case where $w \neq \infty$. For this, we exploit the fact that Möbius transformations act transitively on the Riemann sphere: any case with $w \neq \infty$ can be transformed into one with the pole at infinity by some Möbius transformation $h: \widehat{\mathbb{C}} \rightarrow \widehat{\mathbb{C}}$ such that $h(w)=\infty$. We already know that the domain $h(\Omega)$ has a unique Green's function $g_{h(\Omega)}$ with pole at infinity; since holomorphic mappings preserve harmonicity, we see that $g_{\Omega}=g_{h(\Omega)} \circ h$ is the Green's function for $\Omega$.

Of course, the definition of the Green's function implies that it is positive. Indeed, for any domain $\Omega$ with Green's function $g_{\Omega}$, then $u(z)=-g_{\Omega}(z, w)$ is subharmonic for any $w$, and so the maximum principle implies that it is strictly negative in $\Omega$. Next, we prove another fundamental result of Green's functions, which concerns their relationship to holomorphic mappings.

Theorem 2.3.11. Let $\Omega_{1}$ and $\Omega_{2}$ be domains in $\widehat{\mathbb{C}}$ with non-polar boundaries. Then, if $f: \Omega_{1} \rightarrow \Omega_{2}$ is a meromorphic map,

$$
g_{\Omega_{2}}[f(z), f(w)] \geq g_{\Omega_{1}}(z, w) .
$$

Proof. First, we treat the case $w \neq \infty$ and $f(w) \neq \infty$. Define

$$
u(z)=g_{\Omega_{1}}(z, w)-g_{\Omega_{2}}[f(z), f(w)]
$$

Since the Green's functions are harmonic outside of their poles, it follows that $u$ is subharmonic in $\Omega_{1} \backslash\{w\}$. Furthermore, we have

$$
u(z)=\log \left|\frac{f(z)-f(w)}{z-w}\right|+O(1)=\log \left|f^{\prime}(w)\right|+O(1)
$$

as $z \rightarrow w$, so $u$ is in fact bounded on $\Omega_{1}$. Finally, since $g_{\Omega_{2}}>0$, we conclude that

$$
\limsup _{z \rightarrow \zeta} u(z) \leq \lim g_{\Omega_{1}}(z, w)=0
$$

for n.e. $\zeta \in \partial \Omega_{1}$, and the maximum principle gives the desired inequality.

The cases with $w=\infty$ or $f(w)=\infty$ easily follow from the previous one by remembering that the Green's function in these cases is actually constructed from a Green's function around infinity.

With this, we conclude our foray into the theory of potentials on the plane. However, since we must deal with rational functions - which live on the Riemann sphere -, we shall need results concerning other kinds of potentials. More specifically, we must deal with potentials on $\widehat{\mathbb{C}}$ and on $\mathbb{C}^{2}$. The basis for those lies on the concept of currents - which, in turn, are "just" embellished forms. Our exposition is drawn from Klimek [Kli92] and Berteloot [Ber11, BF11].

First, consider the differential operators of the Riemann sphere:

$$
\begin{aligned}
\frac{\partial}{\partial z} & =\frac{1}{2}\left(\frac{\partial}{\partial x}-i \frac{\partial}{\partial y}\right) \\
\frac{\partial}{\partial \bar{z}} & =\frac{1}{2}\left(\frac{\partial}{\partial x}+i \frac{\partial}{\partial y}\right)
\end{aligned}
$$

As we did for potentials in the plane, these can be used to differentiate distributions supported on the Riemann sphere. If $T: C^{\infty}(\widehat{\mathbb{C}}) \rightarrow \mathbb{R}$ is a distribution, we define its derivatives to be the distributions $\partial T$ and $\bar{\partial} T$ satisfying

$$
\begin{aligned}
& \partial T(f)=T\left(\frac{\partial f}{\partial z}\right) \\
& \bar{\partial} T(f)=T\left(\frac{\partial f}{\partial \bar{z}}\right)
\end{aligned}
$$


for every test function $f: \widehat{\mathbb{C}} \rightarrow \mathbb{R}$. If we also recall the differential forms $d z=d x+i d y$ and $d \bar{z}=d x-i d y$, we can define currents as follows.

Definition. A current of bidegree $(p, q)$ on $\widehat{\mathbb{C}}$ is a $(p, q)$ differential form with coefficients in a space of distributions. The space of all $(p, q)$ currents is denoted by $\mathcal{D}^{p, q}(\widehat{\mathbb{C}})$.

Since distributions and forms are both vector spaces, it is clear that $\mathcal{D}^{p, q}(\widehat{\mathbb{C}})$ is a vector space for any $(p, q)$. Also, since there are no forms higher than $(1,1)$ on the Riemann sphere, it stands to reason that there are also no currents of such bidegrees. The derivative operators for distributions give us a way to transition between different spaces, with some additional care for the forms. If, for a smooth function $f: \widehat{\mathbb{C}} \rightarrow \mathbb{R}$, we have

$$
d f=\frac{1}{2}\left(\frac{\partial}{\partial x}-i \frac{\partial}{\partial y}\right) d z+\frac{1}{2}\left(\frac{\partial}{\partial x}+i \frac{\partial}{\partial y}\right) d \bar{z}
$$

and we want it to be

$$
d f=\partial f d z+\bar{\partial} d \bar{z},
$$

then we must have $\partial=(\partial / \partial z) d z$ and $\bar{\partial}=(\partial / \partial \bar{z}) d \bar{z}$. Thus, the differentials act on $\mathcal{D}^{1,0}(\widehat{\mathbb{C}})$ and $\mathcal{D}^{0,1}$ in the following manner.

$$
\begin{aligned}
\partial(A d \bar{z}) & =\frac{\partial A}{\partial z} d z d \bar{z}=-2 i \frac{\partial A}{\partial z} d x d y \\
\bar{\partial}(B d \bar{z}) & =\frac{\partial B}{\partial \bar{z}} d \bar{z} d z=2 i \frac{\partial B}{\partial \bar{z}} d x d y
\end{aligned}
$$

All in all, we obtain the following diagram - which is not commutative, since $\partial \bar{\partial}=-\bar{\partial} \partial$.

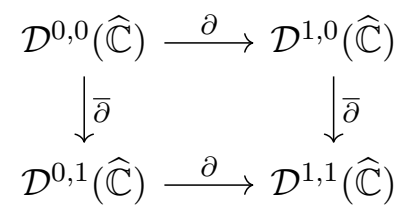

Another operator that we focus on can be derived from the ones above. It is called the MongeAmpère operator, and we define it as

$$
d d^{c}=2 i \partial \bar{\partial} .
$$

Note that this operator acts on $\mathcal{D}^{0,0}(\widehat{\mathbb{C}})$ by sending it straight to $\mathcal{D}^{1,1}(\widehat{\mathbb{C}})$.

There is something else that we must note about currents: as we saw above, every non-negative distribution $T$ gives rise to an associated measure $\mu$. Thus, certain currents can actually be regarded as having measure coefficients. In particular, we have the following.

Proposition 2.3.7. If $u: \widehat{\mathbb{C}} \rightarrow[-\infty, \infty)$ is subharmonic, then $d d^{c} u$ is a measure.

Proof. The proof consists of showing that $d d^{c} u$ is a non-negative distribution. This, in turn, relies on the fact that the Monge-Ampère operator is morally a Laplacian; given coordinates $x, y$ on the Riemann sphere, it becomes

$$
d d^{c}=2 i \partial \bar{\partial}=2 i \frac{1}{4}\left(\frac{\partial}{\partial x}-i \frac{\partial}{\partial y}\right)\left(\frac{\partial}{\partial x}+i \frac{\partial}{\partial y}\right) d z d \bar{z}=\frac{i}{2}\left(\frac{\partial^{2}}{\partial x^{2}}+\frac{\partial^{2}}{\partial y^{2}}\right)(-2 i d x d y)=\nabla^{2} d x d y,
$$

which is a Laplacian operator.

With this in mind, if $u$ is a class $C^{2}$ subharmonic function, then $\nabla^{2} u \geq 0$ and it follows that $d d^{c} u$ is a non-negative distribution. If $u$ is not smooth enough, we shall - as before - approximate it by a sequence of smooth subharmonic functions $u_{n}$ such that $u_{1} \geq u_{2} \geq \cdots \geq u$. Then, by the dominated convergence theorem,

$$
\nabla^{2} u_{n}(\varphi)=\int_{\widehat{\mathbb{C}}} u_{n}(z) \nabla^{2} \varphi(z) d \omega(z) \rightarrow \int_{\widehat{\mathbb{C}}} u(z) \nabla^{2} \varphi(z) d \omega(z)=\nabla^{2} u(\varphi)
$$


for every smooth $\varphi: \widehat{\mathbb{C}} \rightarrow \mathbb{R}$ (here and throughout, $\omega$ denotes the standard area form on the Riemann sphere). Since each $\nabla^{2} u_{n}$ is non-negative, the conclusion follows.

Our last order of business is to expose the generalisation of subharmonic functions to $\mathbb{C}^{2}$.

Definition. A map $u: \Omega \subset \mathbb{C}^{2} \rightarrow[-\infty, \infty)$ is said to be plurisubharmonic - often denoted p.s.h. if it is upper semicontinuous and the map $u \circ \phi$ is subharmonic for every holomorphic map $\phi: \mathbb{D} \rightarrow \Omega$.

Since the definition is based on subharmonic functions and pulling back by holomorphic maps of the disk, p.s.h. functions share many common properties with subharmonic ones - like the sub-mean inequality and the maximum modulus principle, for instance. It also follows that a $C^{2}$ function $u$ is p.s.h. if, and only if, its second derivatives

$$
\frac{\partial^{2}}{\partial z_{i} \partial \bar{z}_{j}}
$$

form a positive semidefinite matrix - the proof is similar to the dimension one case, and we omit it. Likewise, it can also be proved that the limit of a sequence of p.s.h. functions is p.s.h., and conversely that every plurisubharmonic function can be approximated by a monotonically decreasing sequence of smooth subharmonic functions A corollary of this result is that the pullback of a p.s.h. function by a holomorphic map $f: \mathbb{C}^{2} \rightarrow \mathbb{C}^{2}$ is p.s.h. Since the proofs are all very similar to the one-dimensional case, we will not discuss them; we refer interested readers to [Kli92]. 


\section{Chapter 3}

\section{Previous Results}

The first results we must review concern the work of Eremenko, Beardon, Steinmetz and others on the symmetries of Julia sets for polynomials, working our way up to the classification of polynomial Julia sets in [SS95]. Then, we move on to rational functions: we go over Levin \& Przytycki's results concerning the maximal entropy measure of rational maps, and Ye's counter-example for Steinmetz's classification of polynomials associated with a Julia set. Finally, we leave symmetries aside for a moment and lay the groundwork for our use of the ergodic potential associated with a rational map.

\subsection{Symmetries in Polynomial Dynamics}

Before we start on Beardon's results, we must make our concepts clearer. A good place to start are the concepts of isometry and symmetry.

Definition. Let $X$ be a Riemann surface with metric $d$. The map $\sigma: X \rightarrow X$ is an isometry if:

(i) It is holomorphic and bijective, with a holomorphic inverse;

(ii) $d[\sigma(x), \sigma(y)]=d(x, y)$ for every $x, y \in X$.

In other words, isometries are distance-preserving automorphisms; clearly, the set of all isometries of $X$ - denoted $\mathcal{I}(X)$ - is a group under function composition. In fact, though we shall not prove it here, $\mathcal{I}(X)$ is guaranteed to be a Lie group [AB15]. Now, what we call symmetries of a set shall be a special subgroup of $\mathcal{I}(X)$.

Definition. Let $S \subset X$ be a non-empty set. The symmetries of $S$ are a subgroup of $\mathcal{I}(X)$ given by

$$
\Sigma(S)=\{\sigma \in \mathcal{I}(X): \sigma(S)=S\}
$$

In the context of Beardon's work, polynomials are regarded as analytic functions from $\mathbb{C}$ into itself. Thus, the natural set of isometries is $\mathcal{I}(\mathbb{C})=\{z \mapsto a z+b:|a|=1, b \in \mathbb{C}\}$. For a polynomial $P: \mathbb{C} \rightarrow \mathbb{C}$, its symmetry group denotes the symmetries of its Julia set, and we represent it by $\Sigma(P)$ in an abuse of notation.

An important point to notice is that, if $\sigma(z) \in \mathcal{I}(\mathbb{C})$ (or indeed, for any Möbius transformation), $\left(\sigma P \sigma^{-1}\right)^{m}=\sigma P^{m} \sigma^{-1}$ for any $m$. Since Möbius transformations are Lipschitz continuous with respect to the chordal metric, this implies that if $\left\{P^{m}\right\}_{m \geq 1}$ is normal on a domain $U$ then $\left\{\left(\sigma P \sigma^{-1}\right)^{n}\right\}_{m \geq 1}$ is normal on $\sigma(U)$. Therefore, the Julia set of $\sigma P \sigma^{-1}$ is precisely $\sigma[J(P)]$, and thus $\Sigma\left(\sigma P \sigma^{-1}\right)=\sigma \Sigma(P) \sigma^{-1}$. Thus, whenever we see fit, we may conjugate $P$ by an isometry and simplify our problem. Now, Beardon's results can be summarised as:

(i) $P$ and $Q$ are polynomials with the same Julia set if, and only if, $P Q=\sigma Q P$ for some $\sigma \in \Sigma(P)$; 
(ii) $\Sigma(P)$ is either trivial, infinite or cyclic finite. In the second case, $P$ is conjugate to $z \mapsto z^{n}$; in the last one, the order of $\Sigma(P)$ is determine by the largest $k$ such that $P(z)=z^{r} \widetilde{P}\left(z^{k}\right)$ for some polynomial $\widetilde{P}$.

The first step to prove these theorems is to place certain restrictions on the possible structures of $\Sigma(P)$ as a subgroup of $\mathcal{I}(\mathbb{C})$.

Lemma 3.1.1. $\Sigma(P)$ is a group of rotations about some point $\zeta \in \mathbb{C}$. It is either trivial, finite cyclic, or the group of all rotations about $\zeta$.

Proof. Every element of $\Sigma(P)$ is either a rotation or a translation; since $J$ is bounded, $\Sigma(P)$ cannot contain any translations (otherwise, iterating the translation indefinitely would mean that $J$ escapes to infinity!). Now, if $\alpha(z)=a_{0}+a_{1} z$ and $\beta(z)=b_{0}+b_{1} z$ are both symmetries of $J$, their commutator $\alpha \beta \alpha^{-1} \beta^{-1}$ is also an element of $\Sigma(P)$. Doing the calculations,

$$
\alpha \beta \alpha^{-1} \beta^{-1}(z)=z+\left(a_{0}-b_{0}+a_{1} b_{0}-a_{0} b_{1}\right),
$$

and since $\Sigma(P)$ does not contain translations, the term in parenthesis must vanish; thus, $\alpha$ and $\beta$ commute, meaning that $\Sigma(P)$ is a group of rotations around a single point $\zeta \in \mathbb{C}$. If we conjugate $P$ so that $\zeta=0$, we conclude that $\Sigma(P)$ is a subgroup of $S^{1}$, and thus is either finite cyclic or dense in $S^{1}$. Now, we need only prove that $\Sigma(P)$ is closed, and the result follows.

To do so, we prove that $S^{1} \backslash \Sigma(P)$ is open. Take $\sigma(z)=e^{i \theta} z$ an isometry of $\mathbb{C}$ that is not a symmetry of $J$. Then, there exists some $z \in J$ such that $\sigma(z) \in F(P)$, and since $F$ is open there is some neighbourhood $U$ containing $z$ such that $U \cap J=\emptyset$. Intersecting $U$ with a circle of radius $|z|$ centred at the origin, we obtain a non-empty interval $\left(\omega_{1}, \omega_{2}\right)$ such that $|z| e^{i t}$ does not belong to $J$ for any $t \in\left(\omega_{1}, \omega_{2}\right)$. Thus, $e^{i(\theta+t)} z$ does not belong to $J$ for any $t \in\left(\omega_{1}, \omega_{2}\right)$, yielding a neighbourhood of $\sigma$ given by $z \mapsto e^{i(\theta+t)} z, t \in\left(\omega_{1}, \omega_{2}\right)$, that does not intersect $\Sigma(P)$. Therefore, $\Sigma(P)$ is closed.

Consider the case where $\Sigma(P)$ is infinite. Still considering that $\zeta=0, J$ consists of a collection of circles centred at the origin, for the orbit of any $z \in J$ under $\Sigma(P)$ is a circle. However, since $J=\partial F_{\infty}$, and $F_{\infty}$ is connected, it must be the case that $J$ is exactly one circle. Assuming that it is the unit circle, we conclude that $P$ is an analytic self-map of the unit disk that preserves its boundary, and so must be a finite Blaschke product. Since all of its poles are at infinity, $P$ must have all of its zeros at the origin, and so $P(z)=z^{n}$.

The first part or (ii) is now proved; all that is left is to work out the order of $\Sigma(P)$ when it is finite. We start by noticing that symmetries of the Julia set preserve not only the Julia set itself, but also the Green's function $g(z, \infty)$ for $F_{\infty}(P)$ with pole at infinity. Indeed, we saw in the previous chapter that the Green's function of a domain $\Omega$ satisfies $g_{\Omega}(z, w)=g_{f(\Omega)}[f(z), f(w)]$ for any conformal mapping $f: \Omega \rightarrow \widehat{\mathbb{C}}$. Since any $\sigma \in \Sigma(P)$ is a rotation about some point in $\mathbb{C}$ and preserves $F_{\infty}$, this becomes $g(z, \infty)=g[\sigma(z), \infty]$ (we have temporarily suppressed the subscript for the domain from our notation for the Green's function). As a consequence, we obtain the following.

Lemma 3.1.2. Let $\Phi: F_{\infty} \rightarrow \mathbb{C} \backslash \overline{\mathbb{D}}$ be the Böttcher map for the superattracting fixed point at infinity of a polynomial $P$, and $\sigma \in \mathcal{I}(\mathbb{C})$. Then, $\sigma \in \Sigma(P)$ if, and only if, it commutes with $\Phi$.

Proof. We may assume, without loss of generality, that $P$ is in normal form - i.e., $P$ is written as

$$
P(z)=z^{n}+a_{n-2} z^{n-2}+\cdots+a_{1} z+a_{0}
$$

(if this is not the case, we can conjugate $P$ by a transformation of the form $T(z)=\alpha z+\beta$ ). Now, by Böttcher's theorem, the function $\Phi$ satisfies $\Phi P(z)=[\Phi(z)]^{n}$ and has a series expansion

$$
\Phi(z)=z+b_{0}+\frac{b_{1}}{z}+\cdots
$$


for $z$ in a neighbourhood of infinity. Furthermore, since $P$ is in normal form, we get that $b_{0}=0$. Consider, then, an element $\sigma(z)=a z+b$ of $\Sigma(P)$. As is well known, the function $\log |\Phi(z)|$ is the Green's function $g(z, \infty)$ for $F_{\infty}$. Indeed, we note that $\log |\Phi(z)|$ satisfies:

(i) It is harmonic on $F_{\infty}$;

(ii) It goes to zero as $z \rightarrow J$;

(iii) $\log |\Phi(z)|=\log |z|+O(1)$ as $z$ goes to infinity.

Thus, by uniqueness of the Green's function, $\log |\Phi(z)|=g(z, \infty)$. We already know that $g$ is invariant under $\sigma$, which in terms of $\Phi$ means that there is some $\lambda \in S^{1}$ such that

$$
\Phi(a z+b)=\lambda \Phi(z) .
$$

By applying the series expansion of $\Phi$, this becomes

$$
a z+b+O(1 / z)=\lambda z+O(1 / z)
$$

which gives us that $a=\lambda$ and $b=0$. These imply that $\Phi(a z)=a \Phi(z)$, which in turn means that $\sigma$ commutes with $\Phi$.

Conversely, if $\Phi \sigma=\sigma \Phi$ for an isometry $\sigma$, then $g$ is invariant under $\sigma$ and hence so is $F_{\infty}$. The fact that $J=\partial F_{\infty}$ completes the proof.

We wish to convert this condition into something that involves $P$ directly. The fact that $\Phi$ conjugates $P$ to $z^{n}$ allows us to obtain the following.

Proposition 3.1.1. Let $\sigma \in \mathcal{I}(\mathbb{C})$. Then, $\sigma \in \Sigma(P)$ if and only if $P \sigma=\sigma^{n} P$.

Proof. We suppose that $P$ is in normal form. Let $\sigma \in \Sigma(P)$, so that (as seen in the proof of Lemma 3.1.2) $\sigma(z)=a z$. We apply $\Phi P(z)=[\Phi(z)]^{n}$ to $a z$ and use Lemma 3.1.2 to obtain

$$
\Phi P(a z)=[\Phi(a z)]^{n}=[a \Phi(z)]^{n}=a^{n} \Phi P(z) .
$$

Applying the series expansion of $\Phi$ around infinity and comparing the coefficients for $z$, this yields that $P(a z)=a^{n} P(z)$, or equivalently, $P \sigma=\sigma^{n} P$.

Now, suppose that $P \sigma=\sigma^{n} P$ for some $\sigma \in \mathcal{I}(\mathbb{C})$. By induction, there exists some $k$ such that, for every $m \geq 1, P^{m} \sigma=\sigma^{k} P^{m}(k$ depends on $m$ ). Indeed, for $m=1$ we obtain $k=n$. Now, for any $m>1$, the induction hypothesis gives us $P^{m} \sigma=P\left(P^{m-1} \sigma\right)=P \sigma^{k} P^{m-1}$. Now, since $\sigma^{k}=\sigma \sigma^{k-1}$, we can use our original hypothesis to shift the $\sigma$ 's "one-by-one" to obtain $P^{m} \sigma=\sigma^{k n} P^{m}$.

Next, since $\sigma$ is an isometry of the plane, it is a rotation about some $\zeta \in \mathbb{C}$. This implies that $\left|P^{m} \sigma(z)-\zeta\right|=\left|\sigma^{k} P^{m}(z)-\zeta\right|=\left|P^{m}(z)-\zeta\right|$ for every $m$. Therefore, $z$ escapes to infinity if and only if $\sigma(z)$ does, which implies that $F_{\infty}$ - and thus $J$ - is invariant under $\sigma$ and $\sigma \in \Sigma(P)$.

Finally, we can complete the proof of Beardon's result (ii).

Theorem 3.1.1. Let $P$ be a polynomial of degree $n \geq 2$, in normal form, and suppose that $\Sigma(P)$ is finite. Then, the order of $\Sigma(P)$ is the largest $m \geq 1$ such that $P$ can be written as $P(z)=z^{r} \widetilde{P}\left(z^{m}\right)$ for some polynomial $\widetilde{P}$.

Proof. For a given $k \geq 2$, let $\sigma(z)=\mu z$ with $\mu=\exp (2 \pi i / k)$. First, if

$$
P(z)=z^{r} \widetilde{P}\left(z^{k}\right)
$$

for a polynomial $\widetilde{P}$ of degree $d$, then $n=r+k d$ and thus $\mu^{n}=\mu^{r}$. Furthermore,

$$
P \sigma(z)=\mu^{r} z^{r} \widetilde{P}\left(\mu^{k} z^{k}\right),
$$


and since $\mu$ is a $k$-th root of unity we get

$$
P \sigma(z)=\mu^{n} z^{r} \widetilde{P}(z)=\mu^{n} P(z)=\sigma^{n} P(z) .
$$

By Proposition 3.1.1, we conclude that $\sigma \in \Sigma(P)$. In particular, this holds for the largest $k$ such that the decomposition of $P$ is possible - and which we called $m$. This implies that $\Sigma(P)$ contains the group of $m$-th roots of unity; by proving that all symmetries in $\Sigma(P)$ are $m$-th roots of unity, we will conclude that the order of $\Sigma(P)$ is exactly $m$.

To that end, suppose $\sigma(z)=\mu z+b \in \Sigma(P)$. Since $P$ is in normal form, we can assume that $b=0$. Then, as $\Sigma(P)$ is - by hypothesis - finite, we conclude that $\mu$ must be of the form $\exp (2 \pi i / k)$ for some integer $k \geq 2$. Now, we write $P$ as $P(z)=z^{r} R(z)$ for a polynomial $R$ satisfying $R(0) \neq 0$. By Lemma 3.1.1,

$$
\mu^{r} R(\mu z)=\mu^{n} R(z)
$$

for every $z \in \mathbb{C}$. In particular, for $z=0, \mu^{n}=\mu^{r}$, hence

$$
R(\mu z)=R(z) .
$$

As a polynomial of degree $d, R$ can be written as $R(z)=b_{d} z^{d}+b_{d-1} z^{d-1}+\cdots+b_{1} z+b_{0}$, and so the expression above becomes

$$
b_{d} \mu^{d} z^{d}+b_{d-1} \mu^{d-1} z^{d-1}+\cdots+b_{1} \mu z+b_{0}=b_{d} z^{d}+b_{d-1} z^{d-1}+\cdots+b_{1} z+b_{0} .
$$

By comparing coefficients, we conclude that whenever $b_{i} \neq 0$ we must have $\mu^{i}=1$. If $j_{1}, j_{2}, \ldots, j_{s}$ are indices such that $b_{j_{i}} \neq 0$ for $1 \leq i \leq s$, this means that $\mu^{j_{i}}=1$. If $m$ is as required in the theorem's statement, then it must be that $m$ is the greatest common divisor of the $j_{i}$ 's. Therefore, there exist natural numbers $q_{i}$ such that $j_{i}=m q_{i}$, and so there exist integers $p_{i}$ such that

$$
\sum_{i=1}^{s} p_{i} j_{i}=m .
$$

Thus,

$$
\mu^{m}=\mu^{p_{1} j_{1}} \mu^{p_{2} j_{2}} \cdots \mu^{p_{s} j_{s}}=1,
$$

meaning that $\mu$ is an $m$-th root of unity and we are done. Of course, we notice in this proof that $R$ is the polynomial $\widetilde{P}$ stated in the theorem.

Beardon's other result concerns the possibility of different polynomials having the same Julia set. It was proved by Julia himself in 1922 that if two polynomials commute, they share their Julia set. Beardon, then, proved that this condition can be loosened to account for symmetries of the Julia set. Before we get there, we need a technical result.

Lemma 3.1.3. Let $P$ and $Q$ be polynomials of degree $\geq 2$. Then, the following are equivalent.

(a) $J(P)=J(Q)$;

(b) $J(P)$ is completely invariant under $Q$;

(c) $F_{\infty}(P)$ is completely invariant under $Q$.

Proof. (a) implies (b) by the complete invariance of the Julia set. Now, suppose that (b) holds. Since $J(P)$ has more than three points, Montel's theorem guarantees that $\left\{Q^{m}\right\}_{m \geq 1}$ is a normal family in $F(P)$. Thus, $F(P) \subset F(Q)$, and in particular $F_{\infty}(P) \subset F_{\infty}(Q)$. Now, suppose that some point $w \in F_{\infty}(Q)$ is not in $F_{\infty}(P)$. In particular, we can take $w \in J(P)=\partial F_{\infty}(P)$, and (b) implies that $Q^{m}(w)$ both converges to infinity and remains in $J(P)$, which is impossible since $J(P)$ is bounded. Therefore, (b) implies (c). 
Finally, (c) implies that $F_{\infty}(P) \subset F_{\infty}(Q)$, and by the definition of $F_{\infty}(Q)$ we get

$$
F_{\infty}(Q)=\bigcup_{m \geq 0} Q^{-m}\left[F_{\infty}(P)\right]=F_{\infty}(P)
$$

where the last equality follows from (c). In particular, $\partial F_{\infty}(Q)=\partial F_{\infty}(P)$ and we obtain (a).

By applying this lemma to $Q=P \sigma$ and $Q=\sigma P$, we conclude that $\sigma \in \Sigma(P) \Leftrightarrow J(P)=$ $J(\sigma P) \Leftrightarrow J(P)=J(P \sigma)$. From all this, we obtain

Theorem 3.1.2. Let $P$ and $Q$ be polynomials of degree at least two. Then, $J(P)=J(Q)$ if and only if $Q P=\sigma P Q$ for some $\sigma \in \Sigma(P)$.

Proof. First, suppose that $Q P=\sigma P Q$ for a symmetry $\sigma$ of $J(P)$. By induction, $Q P^{n}=(\sigma P)^{n} Q$ and also, since $\sigma \in \Sigma(P)$, we get that $F_{\infty}(P)=F_{\infty}(\sigma P)$. Now, we shall prove that $F_{\infty}(P)$ is invariant under $Q$ and so by Lemma 3.1.3 $J(P)=J(Q)$. To this end, take $z \in F_{\infty}(P)$. By definition, this means that $P^{n}(z) \rightarrow \infty$ as $n \rightarrow \infty$, which also means that $Q P^{n}(z) \rightarrow \infty$ as $n \rightarrow \infty$ since $Q$ is a polynomial. Since $Q P^{n}=(\sigma P)^{n} Q$, we conclude that $(\sigma P)^{n} Q(z)$ also escapes to infinity, and so $Q(z) \in F_{\infty}(\sigma P)=F_{\infty}(P)$. This concludes the first part.

To prove the converse (which was done by Baker and Eremenko in 1987), assume that $J(P)=$ $J(Q)$ and consider the Green's function $g(z, \infty)$ for $F_{\infty}$. Furthermore, assume without loss of generality that $P(z)=a z^{n}+a_{n-2} z^{n-2}+\cdots a_{1} z+a_{0}$ and $Q(z)=b z^{m}+b_{m-2} z^{m-2}+\cdots+b_{1} z+b_{0}$ - this can be done due to the fact that $J(P)=J(Q)$, which means that $\Sigma(P)=\Sigma(Q)$ and in particular both polynomials have the same centre of rotation for their symmetries. Since the Green's function is unique, this implies that

$$
g(z, \infty)=\log |\Phi(z)|+\frac{1}{n-1} \log |a|=\log |\Psi(z)|+\frac{1}{m-1} \log |b|,
$$

where $\Phi$ and $\Psi$ are the Böttcher functions for $P$ and $Q$, respectively, and the constant terms come from the fact that the polynomials were not assumed to be monic. Since $g(z, \infty)$ is related to the equilibrium potential $p_{\mu}$ of $J(P)$, we get that

$$
-I(\mu)=\frac{1}{n-1} \log |a|=\frac{1}{m-1} \log |b|,
$$

whence $b a^{m}=\nu a b^{n}$ for some $\nu \in S^{1}$. Now, from the series expansions of $\Phi$ and $\Psi$ at infinity, we return to the previous equality to conclude that $\Phi \equiv \Psi$, and therefore $\Phi Q=b \Phi^{m}$. Since we already knew that $\Phi P=a \Phi^{n}$, we can write

$$
\Phi(Q P)=b \Phi^{m} P=b a^{m} \Phi^{m n}=\nu a\left(b \Phi^{m}\right)^{n}=\nu a(\Phi Q)^{n}=\nu \Phi(P Q) .
$$

Comparing both ends of this equality in terms of the series expansion of $\Phi$, we conclude that $Q P=\nu P Q$. All that is left is to show that $\sigma(z)=\nu z$ is a symmetry of $J(P)$. We return to the equality $\Phi(Q P)=\nu \Phi(P Q)$, and substitute $Q P=\nu P Q$ and $P Q=w$ to obtain $\nu \Phi(w)=\Phi(\nu w)$, and therefore $\Phi \sigma=\sigma \Phi$. By Lemma 3.1.2, we are done.

Beardon's results explain, among other things, why all quadratic polynomials $P_{c}(z)=z^{2}+c$ have rotational symmetries of order two for their Julia set, and also why no two distinct values of $c$ generate the same Julia set (see Figure 3.1). Soon after, Schmidt and Steinmetz built on his theorems to describe all polynomials that share a Julia set.

More specifically, they provided a way to do that as long as the Julia set involved is not smooth. Below, we state their theorem precisely and prove it.

Theorem 3.1.3. Let $J$ be a Julia set of some polynomial, and assume that it is neither a circle nor a straight line segment. Then, there exists a polynomial $P$ such that any polynomial $Q$ with $J(Q)=J$ satisfies $Q=\sigma P^{n}$, where $\sigma \in \Sigma(P)$ and $n$ is a positive integer. 


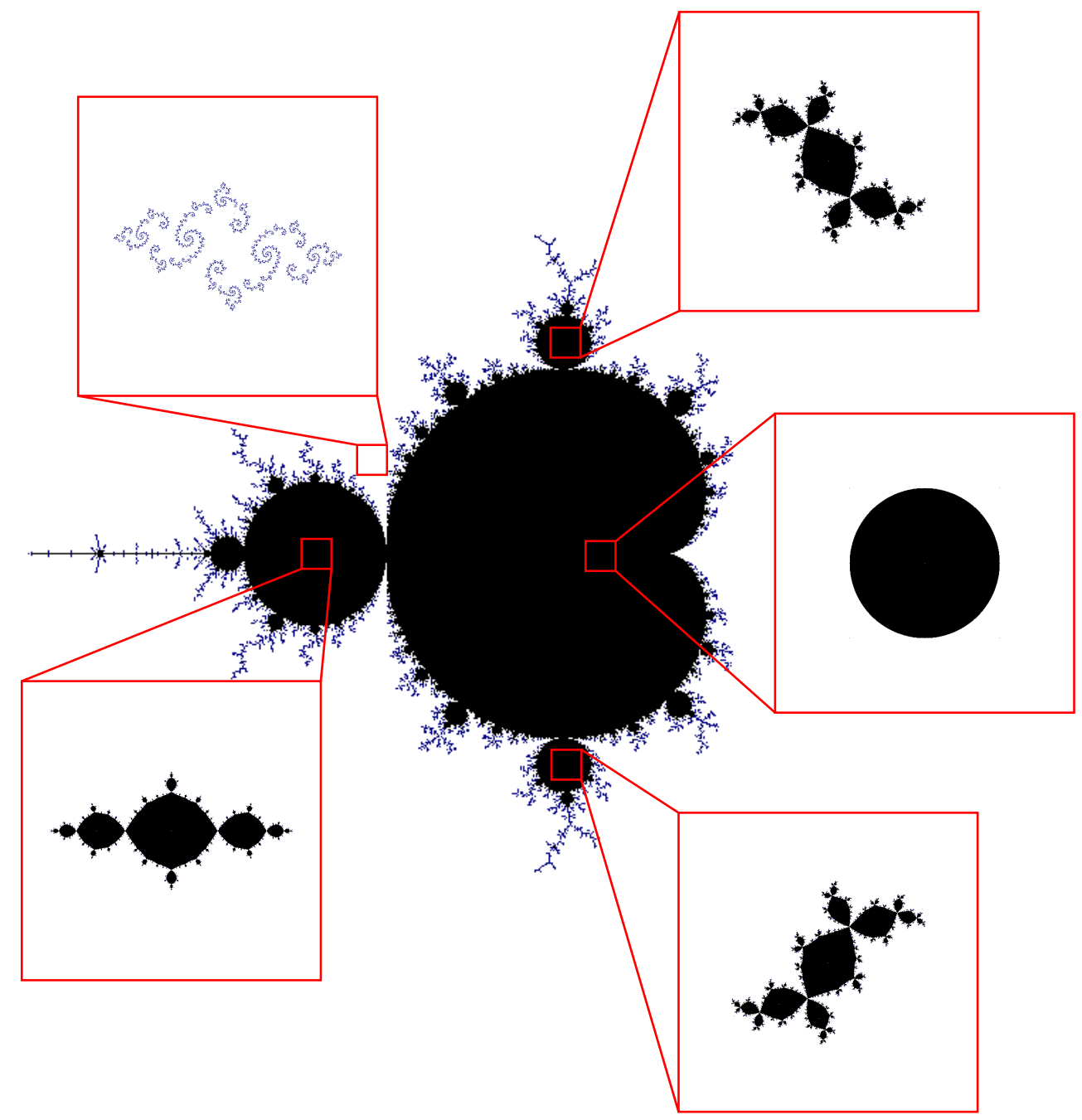

Figure 3.1: The figure shows the infamous Mandelbrot set, with highlights for the filled Julia sets of certain values of $c$.

Proof. Since the degree of a polynomial is bounded from below, there must exist a polynomial $P$ of lowest degree associated with $J$-i.e., such that $J(P)=J$. We assume, without loss of generality, that $P$ is in normal form, and so

$$
P(z)=z^{d}+a_{d-2} z^{d-2}+\cdots+a_{1} z+a_{0}
$$

Since $J$ is assumed not to be a circle, we know that $\Sigma(P)$ has finite order $m$. By Theorem 3.1.1, this implies that $P(z)=z^{r} P_{0}\left(z^{m}\right)$ for a polynomial $P_{0}$. Since any other polynomial $Q$ with $J(Q)=J$ has the same symmetry group, they also admit the same decomposition $Q(z)=z^{s} Q_{0}\left(z^{m}\right)$. Also, by Theorem 3.1.2, $Q P=\sigma P Q$ where $\sigma^{m}=1$, and from these we can deduce that the polynomials

$$
\widehat{P}(z)=z^{r}\left[P_{0}(z)\right]^{m} \quad \text { and } \quad \widehat{Q}(z)=z^{s}\left[Q_{0}(z)\right]^{m}
$$

commute. Our hypothesis that $J$ is not smooth combines with results from Fatou, Julia and Ritt [Fat23, Jul22, Rit20] to conclude that $\widehat{P}$ and $\widehat{Q}$ share an iterate - i.e., there exist integers $k$ and $l$ such that $\widehat{P}^{k}=\widehat{Q}^{l}$. It follows that $(\operatorname{deg} \widehat{P})^{k}=(\operatorname{deg} \widehat{Q})^{l}$, and since $\operatorname{deg} \widehat{P}=\operatorname{deg} P=d$ and $\operatorname{deg} \widehat{Q}=\operatorname{deg} Q$ we conclude that $d$ divides $\operatorname{deg} Q-$ which means that $Q(z)=c z^{k d}+\ldots$ Let $\Phi$ be the (normalised) Böttcher function for $P$ - i.e., $\Phi[P(z)]=[\Phi(z)]^{d}$. As $g(z, \infty)=\log |\Phi(z)|$ in a neighbourhood of infinity, the uniqueness of the Green's function for a domain implies that $Q$ also 
has $\Phi$ as a Böttcher function, since $F_{\infty}(Q)=F_{\infty}(P)$. Now, define a function $r$ by the relation

$$
\Phi[r(z)]=c[\Phi(z)]^{k} .
$$

Then, we have

$$
\Phi[r P(z)]=c[\Phi P(z)]^{k}=c[\Phi(z)]^{k d}=\Phi[Q(z)],
$$

and so $r \circ P=Q$. Writing $r(z)=r_{0}(z)+O(1 / z)$ as $z \rightarrow \infty$, with $r_{0}$ a polynomial (the principal part of $r$ at infinity), we obtain

$$
Q(z)-r_{0} P(z)=O\left(z^{-d}\right) .
$$

It follows that $r=r_{0}$, and so $r$ is a polynomial. Since $r P=Q$, we must have that $J$ is invariant under $r$. Therefore, either $r$ has Julia set $J$ - if $\operatorname{deg} r \geq 2-$ or $r$ is a symmetry of $J$ if $\operatorname{deg} r=1$. In the latter case, we are done; in the former, we apply the same reasoning to $r$ and eventually conclude that $Q=\mu P^{n}$, where $\mu^{d}=1$ and $z \mapsto \mu z$ is a symmetry of $J$.

As a remark, another way of stating Theorem 3.1.3 is as follows. Given a polynomial Julia set $J$, there exists some polynomial $P$ such that the set of all polynomials with Julia set $J$ can be written as

$$
\left\{z \mapsto \sigma P^{n}(z): \sigma \in \Sigma(P), n \geq 1\right\} .
$$

This result came as the apex of all efforts on the problem of polynomials associated with a Julia set. The equivalent problem for rational functions, however, is still open. In the next section, we describe some partial answers resulting from an ergodic-theoretic approach.

\subsection{The Maximal Entropy Measure for Rational Functions}

Let $R: \widehat{\mathbb{C}} \rightarrow \widehat{\mathbb{C}}$ be a rational function of degree $d \geq 2$, and denote by $M_{R}(\widehat{\mathbb{C}})$ the subset of $\mathcal{P}(\widehat{\mathbb{C}})$ of all $R$-invariant Borel probability measures. Since $\widehat{\mathbb{C}}$ is compact, it is a fact that $M_{R}(\widehat{\mathbb{C}})$ is also compact in the weak* topology. Furthermore, it was proved by Lyubich that the measure-theoretic entropy $h_{\mu}(R)$ is upper semi-continuous as a function of the measure $\mu \in M_{R}(\widehat{\mathbb{C}})$ [Lyu83], and we conclude that every rational function has maximal entropy measures - recall that every upper semi-continuous function on a compact set attains its maximum by Proposition 2.3.1. The fact that this measure is unique - along with a way to obtain it - was proved independently by Freire, Lopes and Mañé [FLM83] and Lyubich himself in 1983. However, since both proofs are quite lengthy, we do not include either; instead, we simply state the theorem.

Theorem 3.2.1. Let $R: \widehat{\mathbb{C}} \rightarrow \widehat{\mathbb{C}}$ be a rational map of degree $d \geq 2$. For any $z \in \widehat{\mathbb{C}}$ which is not an exceptional point of $R$, the measures

$$
\mu_{n}=\frac{1}{d^{n}} \sum_{R^{n}(w)=z} \delta_{w}
$$

converge weakly to an ergodic measure $\mu_{R}$ as $n \rightarrow \infty$. Furthermore, $\mu_{R}$ is the unique measure of maximal entropy for $R$.

Remark 3.2.1. It was already pointed out by Brolin in 1965 [Bro65] that the sequence of measures defined above converges to an ergodic measure supported on $J$ in the polynomial case. He also showed that this limit is the equilibrium measure for the Julia set (see Section 2.3 and the discussion below).

Remark 3.2.2. We will always denote the maximal entropy measure of a rational map $R$ by $\mu_{R}$. The equilibrium measure of its Julia set $J(R)$ will be denoted by $\mu_{J}$.

This measure, unlike the equilibrium measure discussed in the previous section, is not uniquely determined by the Julia set - although it is always the case that supp $\mu_{R}=J(R)$. Nonetheless, 
results by Levin and Przytycki [LP97] and - more recently - Ye [Ye15] showed that in some cases it is possible to associate it to both the structure of $J(R)$ and the problem of commuting functions. Their work calls attention to a particular subset of the rational functions - the non-exceptional ones.

Definition. A rational map $R: \widehat{\mathbb{C}} \rightarrow \widehat{\mathbb{C}}$ is said to be non-exceptional if its Julia set is not the whole of $\widehat{\mathbb{C}}$, a circle or an arc.

It is proved in [EvS10], in fact, that these are all possible cases for which the Julia set is smooth. So, in other words, a rational map is exceptional if and only if its Julia set is smooth. Then, Levin and Przytycki's result becomes:

Theorem 3.2.2. Let $R$ and $S$ be non-exceptional rational maps of degree $\geq 2$. Then, the following are equivalent.

(i) $\mu_{R}=\mu_{S}$;

(ii) There exist iterates $\widehat{R}$ of $R$ and $\widehat{S}$ of $S$ such that, for some natural numbers $M$ and $N$, it holds:

$$
\left(\widehat{S}^{-1} \circ \widehat{S}\right) \circ \widehat{S}^{M}=\left(\widehat{R}^{-1} \circ \widehat{R}\right) \circ \widehat{R}^{N},
$$

where $\widehat{S}^{-1} \circ S$ and $\widehat{R}^{-1} \circ R$ denote some single-valued function obtained by analytic continuation of a branch.

If, in addition, neither $R$ nor $S$ have any parabolic or rotation domains, then $\mu_{R}=\mu_{S}$ if and only if $J(R)=J(S)$.

The proof is quite technical, and we will not dwell on it. Suffice to say that it relies on the fact that $\mu_{R}$ is the unique balanced measure for $R$-i.e., the only measure for which $\mu_{R}[R(A)]=d \mu_{R}(A)$ for every measurable set $A$ where $R$ is injective. This, in turn, was proved in [FLM83].

This result is very close to what we aim to do here. Despite its limitations - which are mostly the fact that it only holds for a restricted subset of the rational functions, and the fact that neither $\mu_{R}$ nor $\widehat{R}$ are readily computable -, it will prove invaluable to us as we attempt to reconstruct something like a Green's function for rational maps.

Lopes proved in 1986 [Lop86] that - under the condition that infinity be a fixed point in the Fatou set $-\mu_{R}=\mu_{J}$ if, and only if, $R$ is a polynomial. Therefore, it follows that for polynomials having equal maximal entropy measures is always equivalent to having the same Julia set regardless of being an exceptional map or not. Furthermore, Schmidt and Steinmetz's Theorem 3.1.3 implies that if $P$ and $Q$ are polynomials with $\mu_{P}=\mu_{Q}$, then there exists $\sigma \in \Sigma(P)$ such that

$$
P^{n}=\sigma Q^{m} \quad \text { for some } m, n \in \mathbb{N} \text {. }
$$

Remarkably, it was shown by Ye in 2015 that this is not always the case for rational functions, even the non-exceptional ones [Ye15]. More specifically, he proved the following and then provided examples of functions satisfying its hypotheses.

Theorem 3.2.3. Suppose $R, S$ and $T$ are rational functions with degrees $\geq 2$, satisfying:

(i) $R \neq \sigma S$, for any $\sigma \in \operatorname{PSL}(2, \mathbb{C})$;

(ii) $T R=T S$.

Then, then functions $F=R T$ and $G=S T$ have $\mu_{F}=\mu_{G}$, and

$$
F^{n} \neq \sigma G^{m}
$$

for every $\sigma \in \operatorname{PSL}(2, \mathbb{C})$ and $m, n \in \mathbb{N}$. 
Proof. Since $T R=T S$, we have $R T \circ R T=R \circ(T R) \circ T=R \circ(T S) \circ T=R T \circ S T$, which means that $F \circ F=F \circ G$. Also, by the same reasoning, $T \circ F^{i}=T \circ G^{i}$ for any $i \geq 1$. Hence, $\left(F^{-1} \circ F\right) F=G$, and so it follows from Theorem 3.2.2 that $\mu_{F}=\mu_{G}$.

Now, assume that $F^{n}=\sigma G^{m}$ for some $m, n \in \mathbb{N}$ and $\sigma \in \operatorname{PSL}(2, \mathbb{C})$. Then, since $\operatorname{deg} F=\operatorname{deg} G$, it follows that $m=n$, and so $F^{n}=\sigma G^{n}$. Thus, since $F^{n}(z)=R T \circ F^{n-1}(z)$ and $G^{n}(z)=$ $S T \circ G^{n-1}(z)$, hypothesis (ii) implies that $F=\sigma G$, which is a contradiction.

Next, we discuss Ye's example - which is actually a whole family of rational functions.

Corollary 3.2.1. For any $a \in \mathbb{C}$, the functions

$$
F(z)=a\left(z^{3}-3 z\right)+\frac{1}{a\left(z^{3}-3 z\right)} \quad \text { and } \quad G(z)=a \mu\left(z^{3}-3 z\right)+\frac{1}{a \mu\left(z^{3}-3 z\right)},
$$

where $\mu$ is any (non-trivial) third root of unity, have the same measure of maximal entropy and satisfy condition (3.2).

Proof. Take $T(z)=z^{3}-3 z, R(z)=a z+(a z)^{-1}$ and $S(z)=a \mu z+(a \mu z)^{-1}$. Then, the fact that $\mu$ is a third root of unity implies that

$$
T S(z)=T R(z)=a^{3} z^{3}+\frac{1}{a^{3} z^{3}}
$$

and also that $R \neq \sigma S$ for any Möbius transformation $\sigma$. The conclusion follows from Theorem 3.2 .3 .

This is not to say, however, that everything is hopeless for rational functions. In the same paper, Ye proved that for most - in some topological sense - rational maps, the measure of maximal entropy is "rigid" (that is, the only rational maps with a certain measure of maximal entropy are given by iterations of a lowest degree map). To see what is meant by "most", consider that a rational function of degree $d$

$$
R(z)=\frac{a_{d} z^{d}+a_{d-1} z^{d-1}+\cdots+a_{0}}{b_{d} z^{d}+b_{d-1} z^{d-1}+\cdots+b_{0}}
$$

can be identified with its coefficient vector $\left(a_{d}, a_{d-1}, \ldots, a_{0}, b_{d}, b_{d-1}, \ldots, b_{0}\right) \in \mathbb{C}^{2 d+2}$. However, not any vector in $\mathbb{C}^{2 d+2}$ gives rise to a rational function of degree $d$; the polynomials $a_{d} z^{d}+a_{d-1} z^{d-1}+$ $\cdots a_{0}$ and $b_{d} z^{d}+b_{d-1} z^{d-1}+\cdots+b_{0}$ must not have any common roots. If we denote these roots by $\lambda_{1}, \lambda_{2}, \ldots, \lambda_{d}$ and $\mu_{1}, \mu_{2}, \ldots, \mu_{n}$ counted with multiplicity, then the roots of the resultant polynomial

$$
\operatorname{res}\left(a_{d}, a_{d-1}, \ldots, a_{0}, b_{d}, \ldots, b_{0}\right)=\Pi_{1 \leq i, j \leq d}\left(\lambda_{i}-\mu_{j}\right)
$$

are precisely the coefficient vectors to be avoided. Therefore, the space Rat ${ }_{d}$ of rational functions of degree $d$ can be seen as the complement of the set of roots of a polynomial in $\mathbb{C}^{2 d+2}$ - in fact, the resultant polynomial is homogeneous, and Rat ${ }_{d}$ passes naturally to the projective space since multiplying all coefficients by a constant causes it to cancel out. Therefore, Rat ${ }_{d}$ is an affine (quasiprojective) variety in $\mathbb{P}^{2 d+1}(\mathbb{C})$. Its Zariski topology ${ }^{1}$ yields what we mean by "most": we define a property to be generic if it holds for a countable intersection of Zariski open sets. Finally, we denote by $M_{R}$ the set of all rational functions with the same measure of maximal entropy as $R$. With this language, Ye's conclusion is the following.

Theorem 3.2.4. For generic rational functions $R \in$ Rat $_{d}$, with $d \geq 2$, we have

(i) $M_{R}=\left\{R^{n}: n \geq 1\right\}$ if $d \geq 3$;

(ii) $M_{R}=\left\{\sigma \circ R^{n}: \sigma \in \operatorname{PSL}(2, \mathbb{C}), R \sigma=R, n \geq 1\right\}$ if $d=2$.

\footnotetext{
${ }^{1}$ This is the topology defined by saying that closed sets are zeroes of polynomials.
} 
This condition is similar to Steinmetz's condition (3.1), but it is remarkably different in that it does not allow for symmetries of the Julia set if $d \geq 3$. Later, we will see how Theorem 3.2.4 shows that generic rational functions might have trivial symmetry groups.

\subsection{A Modified Green's Function for Rational Maps}

In this section, we call on pluripotential theory to obtain, given a rational map $R$, some function $g_{R}: \widehat{\mathbb{C}} \rightarrow \mathbb{R}$ with enough properties of the original Green's function to be of help. Admittedly, the results are less than ideal; this "Green's function" does not depend solely on the structure of the Julia set, and it is not harmonic at all - merely continuous. Nonetheless, it will give us precious insights into the necessary conditions for symmetries. Most of the work in this section can be found in [Ber11].

Firstly, we shall leave the Riemann sphere and rational functions behind. Given a rational map $R: \widehat{\mathbb{C}} \rightarrow \widehat{\mathbb{C}}$ of degree $d \geq 2$, we shall lift it to a homogeneous polynomial $\widehat{R}: \mathbb{C}^{2} \rightarrow \mathbb{C}^{2}$ and work from there. So, suppose that $R(z)=P(z) / Q(z)$ where $P: \mathbb{C} \rightarrow \mathbb{C}$ and $Q: \mathbb{C} \rightarrow \mathbb{C}$ are co-prime polynomials. If $\pi: \mathbb{C}^{2} \backslash\{(0,0)\}$ given by $\pi\left(z_{1}, z_{2}\right)=z_{1} / z_{2}$ is the standard projection of $\mathbb{C}^{2}$ onto the Riemann sphere, then defining

$$
\widehat{R}\left(z_{1}, z_{2}\right)=z_{2}^{d}\left(P\left(z_{1} z_{2}^{-1}\right), Q\left(z_{1} z_{2}^{-1}\right)\right)
$$

yields immediately that the following diagram commutes.

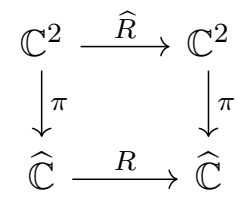

Since $\widehat{R}$ is a homogeneous polynomial, we can guarantee that the sequence

$$
G_{n}\left(z_{1}, z_{2}\right):=\frac{1}{d^{n}} \log \left\|\widehat{R}^{n}\left(z_{1}, z_{2}\right)\right\|
$$

converges uniformly on compact subsets of $\mathbb{C}^{2} \backslash\{(0,0)\}$. Indeed, since $\widehat{R}$ is a homogeneous polynomial, given any compact subset of $\mathbb{C}^{2} \backslash\{(0,0)\}$ there exists some $M \geq 1$ such that

$$
\frac{1}{M}\left\|\left(z_{1}, z_{2}\right)\right\|^{d} \leq\left\|\widehat{R}\left(z_{1}, z_{2}\right)\right\| \leq M\left\|\left(z_{1}, z_{2}\right)\right\|^{d} .
$$

Thus, $M^{-1}\left\|\widehat{R}^{n}\left(z_{1}, z_{2}\right)\right\|^{d} \leq\left\|\widehat{R}^{n+1}\left(z_{1}, z_{2}\right)\right\| \leq M\left\|\widehat{R}^{n}\left(z_{1}, z_{2}\right)\right\|$, and so $\left|G_{n+1}\left(z_{1}, z_{2}\right)-G_{n}\left(z_{1}, z_{2}\right)\right| \leq$ $d^{-(n+1)}(\log M)$ and convergence is uniform. We define the function

$$
G_{\widehat{R}}:=\lim _{n \rightarrow \infty} G_{n} .
$$

Since it is the uniform limit of a sequence of continuous - except for a pole at the origin - plurisubharmonic functions, it is plurisubharmonic and continuous with a single pole. Furthermore, its definition implies that $G_{\widehat{R}} \circ \widehat{R}=d \cdot G_{\widehat{R}}$. If we can pass it to the Riemann sphere, we will obtain a continuous function which is bound to have some relevant dynamical properties. This motivates the following definition.

Definition. The Green's function of a rational map $R$ is the function $g_{R}: \widehat{\mathbb{C}} \rightarrow \mathbb{R}$ given by

$$
G_{\widehat{R}}\left(z_{1}, z_{2}\right)-\log \left\|\left(z_{1}, z_{2}\right)\right\|=g_{R} \circ \pi\left(z_{1}, z_{2}\right) .
$$

Notice that, by the definition of $G_{\widehat{R}}, G_{\widehat{R}}\left(\lambda z_{1}, \lambda z_{2}\right)=G_{\widehat{R}}\left(z_{1}, z_{2}\right)+\log |\lambda|$; therefore, $g_{R}$ is actually well-defined by the formula above. So, we obtain a function on $\widehat{\mathbb{C}}$ that comes from the dynamical 
properties of $R$. It is, by construction, continuous We also get something else: a relation between $g_{R}$ and $\mu_{R}$, the unique maximal entropy measure.

Theorem 3.3.1. For any rational map $R, g_{R}$ satisfies $\mu_{R}=\omega+d d^{c} g_{R}$, where $\omega$ denotes the standard (normalised) area form on $\widehat{\mathbb{C}}$.

Proof. Stokes' theorem implies that

$$
\int_{\widehat{\mathbb{C}}} d d^{c} g_{R}=\int_{\partial \widehat{\mathbb{C}}} d^{c} g_{R}=0,
$$

and so $\omega+d d^{c} g_{R}$ is indeed a probability measure since $\int_{\widehat{\mathbb{C}}} \omega=1$. The rest of the proof - which we will not give here, but can be found in [Ber11] - hinges on proving that $\omega+d d^{c} g_{R}$ is a balanced measure supported on $J(R)$.

Theorem 3.3.1 is sometimes stated as saying that $g_{R}$ is a potential for the measure $\mu_{R}$. Notice that it does not resemble the potential we defined for measures on the plane. That would be the following definition.

Definition. The elliptic potential associated to a probability measure $\mu$ on the Riemann sphere is

$$
u_{\mu}(z)=\int_{\widehat{\mathbb{C}}} \log \frac{1}{\rho(z, w)} d \mu(w)
$$

where $\rho$ denotes the chordal distance on $\widehat{\mathbb{C}}$.

The convention of using the inverse of the distance instead of simply the distance is due to the fact that $\log \rho$ is not harmonic; it is superharmonic, and so we invert the metric in order to make $u_{R}$ a subharmonic function. If $R$ is a rational map of degree $\geq 2$, we can choose $\mu_{R}$ as our measure and define the ergodic potential of $R$, written as $u_{R}$ by an abuse of notation. The most important (at least to this work) facts about $u_{R}$ were pointed out by Okuyama in [Oku05] (we give a slightly modified statement and proof).

Proposition 3.3.1. For any rational map $R$ of degree greater than one, $u_{R}$ is continuous and equal up to an additive constant to $-g_{R}$.

Proof. For the logarithm, we have

$$
d d^{c} \log \frac{1}{\rho(\cdot, z)}=\omega-\delta_{z}
$$

Integrating with respect to $\mu_{R}$ yields

$$
d d^{c} u_{R}=\omega-\mu_{R},
$$

and since $\mu_{R}=\omega+d d^{c} g_{R}$ this becomes

$$
d d^{c}\left(u_{R}+g_{R}\right)=0
$$

This implies that the sum $u_{R}+g_{R}$ is harmonic throughout $\widehat{\mathbb{C}}$. Since the only harmonic functions on the Riemann sphere are constants (a consequence of the maximum modulus principle), it follows that $u_{R}=-g_{R}+c$, where $c \in \mathbb{R}$ is a constant. As $g_{R}$ is continuous, the conclusion follows.

Proposition 3.3.1 is the last previous result of importance to us. Now, we are ready to state and prove our own conclusions. 


\section{Chapter 4}

\section{Results}

\subsection{General Considerations}

In Section 3.1, we considered polynomials as functions of the complex plane $\mathbb{C}$ onto itself. Now, doing so for general rational functions has the considerable disadvantage that the point at infinity has, in general, pre-images distinct from itself. Therefore, it is best to consider rational maps as $R: \widehat{\mathbb{C}} \rightarrow \widehat{\mathbb{C}}$, and assume the corresponding group of isometries $\mathcal{I}(\widehat{\mathbb{C}})$. First, we point out some properties of this new isometry group.

The holomorphic automorphisms of the Riemann sphere are the Möbius transformations

$$
z \mapsto \frac{a z+b}{c z+d},
$$

with $a, b, c, d \in \mathbb{C}$ and $a d-b c \neq 0$. Since multiplying all coefficients by the same constant does not change the transformation, we can assume that $a d-b c=1$. Therefore, we can see that the transformation

$$
\left(\begin{array}{ll}
a & b \\
c & d
\end{array}\right) \mapsto \frac{a z+b}{c z+d}
$$

maps the group $\operatorname{SL}(2, \mathbb{C})$ of two-by-two complex matrices with determinant one into the group $\mathcal{M}(\widehat{\mathbb{C}})$ of Möbius transformations. A quick computation show that it is a homomorphism, and that its kernel is $\{I,-I\}-$ where $I$ is the identity matrix. Therefore, the group $\operatorname{PSL}(2, \mathbb{C})$ consisting of $\mathrm{SL}(2, \mathbb{C})$ modulo the subgroup $\{I,-I\}$ is isomorphic to $\mathcal{M}(\widehat{\mathbb{C}})$, and the isomorphism is realised in a very intuitive way. In particular, this implies that $\mathcal{M}(\widehat{\mathbb{C}})$ is a complex Lie group of dimension three, and it is neither compact nor simply connected.

The isometry group $\mathcal{I}(\widehat{\mathbb{C}})$ is a subgroup of $\mathcal{M}(\widehat{\mathbb{C}})$. Since it is the isometry group of a smooth manifold, it is itself a Lie group and thus is a Lie subgroup of the Möbius transformations. In fact, we can give an explicit description of the elements of $\mathcal{I}(\widehat{\mathbb{C}})$; since every isometry must preserve the chordal metric element given by

$$
\frac{2|d z|}{1+|z|^{2}}
$$

straightforward computation shows that the isometries of the Riemann sphere can be described as

$$
\mathcal{I}(\widehat{\mathbb{C}})=\left\{z \mapsto \frac{a z-\bar{b}}{b z+\bar{a}}:|a|^{2}+|b|^{2}=1\right\} .
$$

Now, consider the natural identification $\Phi: \widehat{\mathbb{C}} \rightarrow S^{2}$ of $\widehat{\mathbb{C}}$ with $S^{2} \subset \mathbb{R}^{3}$. By construction, the chordal metric $\rho$ is the restriction to $S^{2}$ of the Euclidean metric of $\mathbb{R}^{3}$. Consequently, every orientationpreserving rotation $H$ of $\mathbb{R}^{3}$ yields an isometry of the Riemann sphere given by $\Phi^{-1} H \Phi$. Likewise, if $\sigma: \widehat{\mathbb{C}} \rightarrow \widehat{\mathbb{C}}$ is an isometry, then $\Phi \sigma \Phi^{-1}$ is an orientation-preserving isometry of $S^{2}$ that can be easily extended to all of $\mathbb{R}^{3}$ as an orthogonal transformation. It follows that $\mathcal{I}(\widehat{\mathbb{C}})$ is isomorphic - as a Lie group - to the group $\mathrm{SO}(3)$ of orientation-preserving orthogonal transformations of $\mathbb{R}^{3}$. Most 
importantly, this implies that $\mathcal{I}(\widehat{\mathbb{C}})$ is a compact and connected but not simply connected real Lie group of dimension three. Also, it must be remarked that $\mathcal{I}(\widehat{\mathbb{C}})$ acts transitively on the Riemann sphere - i.e., for every $z, w \in \widehat{\mathbb{C}}$ there exists some $\sigma \in \mathcal{I}(\widehat{\mathbb{C}})$ such that $\sigma(z)=w$. This can also be stated as saying that $\widehat{\mathbb{C}}$ is a homogeneous space.

Finally, given a rational map $R$ with Julia set $J(R)$, we can put our first restrictions on the structure of its symmetry group

$$
\Sigma(R)=\{\sigma \in \mathcal{I}(\widehat{\mathbb{C}}): \sigma[J(R)]=J(R)\} .
$$

Lemma 4.1.1. $\Sigma(R)$ is a closed set.

Proof. If $J(R)=\widehat{\mathbb{C}}$, then every isometry of $\widehat{\mathbb{C}}$ preserves $J(R)$. Hence, $\Sigma(R)=\mathcal{I}(\widehat{\mathbb{C}})$, which is a closed group, and the conclusion follows.

If $J(R) \neq \widehat{\mathbb{C}}$, we cannot have $\Sigma(R)=\mathcal{I}(\widehat{\mathbb{C}})$ because the latter acts transitively on the Riemann sphere. Thus, we consider $\sigma \in \mathcal{I}(\widehat{\mathbb{C}}) \backslash \Sigma(R)$. We know that there exists some $z \in J(R)$ such that $\sigma(z) \in F(R)$, which is an open set. Therefore, there must be a neighbourhood $U$ of $\sigma(z)$ that does not intersect $J(R)$. This yields a neighbourhood $V \subset \mathcal{I}(\widehat{\mathbb{C}})$ such that $V \sigma \cap \Sigma(R)=\emptyset$ by the following construction. For every $w \in U$, the homogeneity of $\widehat{\mathbb{C}}$ implies the existence of some $\gamma \in \mathcal{I}(\widehat{\mathbb{C}})$ such that $\gamma[\sigma(z)]=w$. Since the isometries act smoothly on the Riemann sphere, the collection of all such $\gamma$ for every $w \in U$ yields a neighbourhood $V$ of the identity - which is also in this collection for $w=\sigma(z)$. By continuity of the group operations, $V \sigma$ is a neighbourhood of $\sigma$ which, by construction, does not intersect $\Sigma(R)$. Thus, $\mathcal{I}(\widehat{\mathbb{C}}) \backslash \Sigma(R)$ is open.

Though simple, this result has crucial consequences. Firstly, as a closed subgroup of a compact set, we get that $\Sigma(R)$ is compact. Secondly, by Cartan's closed subgroup theorem, it follows that $\Sigma(R)$ is a Lie subgroup of $\mathcal{I}(\widehat{\mathbb{C}})$ - which means that it is an embedded submanifold of $\mathcal{I}(\widehat{\mathbb{C}})$. Hence, we obtain our first serious restriction on the structure of $\Sigma(R)$.

Theorem 4.1.1. For any rational map $R, \Sigma(R)$ is (isomorphic to) one of:

(i) The trivial group;

(ii) A group of roots of unity;

(iii) A dihedral group generated by a root of unity $z \mapsto e^{2 \pi i / k} z$ and an inversion $z \mapsto 1 / z$;

(iv) The orientation-preserving symmetries of a regular tetrahedron, octahedron or icosahedron;

(v) $S[O(1) \times O(2)]$ - i.e., the group of isometries of the form $z \mapsto e^{i \theta} z$ and $z \mapsto e^{i \theta} / z$ for any $\theta \in[0,2 \pi)$

(vi) All isometries of the Riemann sphere.

Proof. We note that there is little to be done in cases (i) and (vi) from a symmetry point of view. Although their dynamics may be interesting - case (vi), for instance, are the Lattès maps -, we assume now that $\Sigma(R)$ is neither trivial nor equal to $\mathcal{I}(\widehat{\mathbb{C}})$.

The first distinction we must make is between a discrete and a continuous symmetry group. In the former, $\Sigma(R)$ must be a discrete Lie subgroup of $\mathcal{I}(\widehat{\mathbb{C}})$. If it is infinite, the compactness of $\mathcal{I}(\widehat{\mathbb{C}})$ implies that it has a cluster point $\sigma \in \mathcal{I}(\widehat{\mathbb{C}})$ - which, since $\Sigma(R)$ is closed, also belongs to $\Sigma(R)$. Around $\sigma$, the structure of $\Sigma(R)$ as an embedded, discrete Lie group breaks down; it follows that if $\Sigma(R)$ is discrete, it must also be finite. Then, the classification of finite subgroups of $\mathrm{SO}(3)$ in [Car12, Theorem 4.1] gives us cases (ii) through (iv) as the only possibilities and this concludes the discrete case.

Suppose now that $\Sigma(R)$ is a continuous Lie subgroup of $\mathcal{I}(\widehat{\mathbb{C}})$. Take the connected component $H$ of $\Sigma(R)$ containing the identity, which is also a Lie subgroup and has an associated Lie subalgebra $\mathfrak{h} \subset \mathfrak{i}(\widehat{\mathbb{C}})$. Since $\mathcal{I}(\widehat{\mathbb{C}})$ is isomorphic to $\mathrm{SO}(3)$, it follows that $\mathfrak{i}(\widehat{\mathbb{C}}) \simeq \mathfrak{s o}(3)$ and we already know 
that the latter is isomorphic to $\mathbb{R}^{3}$ with the Lie algebra structure given by the vector product. Therefore, $\mathfrak{i}(\widehat{\mathbb{C}}) \simeq \mathbb{R}^{3}$ and so $\mathfrak{h}$ is conjugate to a Lie subalgebra of $\mathbb{R}^{3}$. However, the only non-trivial Lie subalgebras of $\mathbb{R}^{3}$ have dimension one; hence, $H$ is a one-dimensional Lie subgroup of $\mathcal{I}(\widehat{\mathbb{C}})$.

Next, we know that $H$ admits a parametrisation through the Lie exponential of $\mathcal{I}(\widehat{\mathbb{C}})$. Thus, we write $H=\{\exp [t X]: t \in \mathbb{R}\}$ for some $X$ in its Lie algebra $\mathfrak{h} \subset \mathfrak{i}(\widehat{\mathbb{C}})$. The action of the isometry group on the Riemann sphere yields a flows $\phi: \mathbb{R} \times \widehat{\mathbb{C}} \rightarrow \widehat{\mathbb{C}}$ defined as $\phi(t, z)=\exp [t X](z)$, which has an associated vector field $\vec{F}: \widehat{\mathbb{C}} \rightarrow T \widehat{\mathbb{C}}$ - called the action field of $H$ - given by

$$
\vec{F}(z)=\partial_{t} \phi(0, z)=\left.\frac{d}{d t} \exp [t X] z\right|_{t=0} .
$$

By the hairy ball theorem, there exists $z_{0} \in \widehat{\mathbb{C}}$ such that $\vec{F}\left(z_{0}\right)=0$; this point satisfies $\phi\left(t, z_{0}\right)=z_{0}$ for all $t$, and so it is fixed by every $\sigma \in H$. As every isometry of $\widehat{\mathbb{C}}$ has exactly two antipodal fixed points, it follows that $z_{0}$ 's antipode is also fixed by every element of $H$. Conjugating the Riemann sphere by an isometry so that $z_{0}=0$, we conclude that $H$ is conjugate to $S^{1}=\left\{e^{i t}: t \in[0,2 \pi)\right\}$.

Now, take a $z \in J(R)$ that is not fixed by the action of $H$. Its orbit under $H$ must be a circle, and so $J(R)$ is a collection of concentric circles - either a single one or an uncountable amount of them. In the former case, $\Sigma(R)$ are the symmetries of a circle, so its elements are either of the form $z \mapsto e^{i \theta} z$ or $z \mapsto e^{i \theta} / z$. We argue that the latter case is not possible. Indeed, the smoothness of each connected component of $J(R)$ implies that all multipliers of periodic orbits are real [Fat20, Mil06a, EvS10] and, following Eremenko and van Strien, this means that $J(R)$ is contained in a single circle - and therefore is a single circle.

Remark 4.1.1. It was shown by Eremenko and van Strien that if $J(R)$ is a circle, then either $R$ or $R^{2}$ is conjugate to a Blaschke product. Thus, a corollary of Theorem 4.1.1 is that if $\Sigma(R)$ is continuous, then $R$ is either a Lattès map or conjugate to a Blaschke product (up to taking the second iterate of $R$ ).

Remark 4.1.2. It should be noted that all cases in Theorem 4.1.1 can actually be realised as symmetry groups of some function. Case (i), in fact, is shown in Theorem 4.1.3 to be the most common. For any rational map $R$, consider the group $\operatorname{Aut}(R):=\{\sigma \in \operatorname{PSL}(2, \mathbb{C}): R \sigma=\sigma R\}$. By Proposition 4.1.1, any $\sigma \in \operatorname{Aut}(R)$ that is also an isometry of the Riemann sphere belongs to $\Sigma(R)$. Now, by a theorem of Doyle and McMullen [DM89], for any finite subgroup $G \subset \operatorname{PSL}(2, \mathbb{C})$ there exists a rational map $R_{G}$ such that $\operatorname{Aut}\left(R_{G}\right)=G$. Therefore, by choosing $G$ as any of cases (ii) (iv) above, we can guarantee the existence of rational maps with any finite symmetry group. For the continuous symmetry groups, case (v) is realised by power maps $z \mapsto z^{ \pm n}$ and case (vi), by the Lattès maps.

Our next task is to find necessary or sufficient conditions for an isometry $\sigma \in \mathcal{I}(\widehat{\mathbb{C}})$ to be a symmetry of a given rational map. We offer one sufficient condition and two necessary ones, and they are partial extensions of Beardon's results for polynomials (see Proposition 3.1.1).

Proposition 4.1.1. Let $R$ be a rational function and $\sigma \in \mathcal{I}(\widehat{\mathbb{C}})$, and suppose that $R$ is not a Lattès map. If $R \sigma=\sigma^{k} R$ for some $k \geq 1$, then $\sigma \in \Sigma(R)$.

Proof. We will show that the Fatou set of $R$ is invariant under $\sigma$. Take $z \in F(R)$. By the ArzelàAscoli theorem, for any $\epsilon>0$ there is a neighbourhood $U$ of $z$ satisfying $\operatorname{diam}\left[R^{m}(U)\right]<\epsilon$ for every $m \geq 1$. Consider, then, how $R$ behaves at $\sigma(z)$. By induction, our hypothesis implies that there exists for all $m \geq 1$ an $l \geq 1$ - which depends on $m$ - such that $R^{m} \sigma=\sigma^{l} R^{m}$. Indeed, for the case $m=1, l$ is easily seen to be equal to $k$ as per our hypothesis. Now, for any $m, R^{m+1} \sigma=R\left(R^{m} \sigma\right)$, and the induction hypothesis gives us $R^{m+1} \sigma=R\left(\sigma^{l} R^{m}\right)$. By using that $R \sigma=\sigma^{k} R$, we can shift the $\sigma$ 's "one-by-one" to obtain $R^{m+1} \sigma=\sigma^{k l} R^{m+1}$. Therefore, $\operatorname{diam}\left[R^{m} \sigma(U)\right]=\operatorname{diam}\left[\sigma^{l} R^{m}(U)\right]$; since $\sigma$ is an isometry of the Riemann sphere, it leaves the diameter of a set unchanged, and thus $\operatorname{diam}\left[R^{m} \sigma(U)\right]=\operatorname{diam}\left[R^{m}(U)\right]$ for every $m \geq 1$. Since the terms on the right-hand side are limited by $\epsilon$, this implies (by the Arzelà-Ascoli theorem) that $R^{m}$ is a normal family at $\sigma(z)$, and thus 
$\sigma[F(R)] \subset F(R)$. Since $\sigma^{-1}$ is also an isometry, we can apply the same reasoning to conclude that $\sigma^{-1}[F(R)] \subset F(R)$, and so $F(R)$ - and thus $J(R)$ - is invariant under $\sigma$ and $\sigma \in \Sigma(R)$.

One of our necessary conditions will allows to specify a value for $k$ in Proposition 4.1.1, even if only for specific situations. In order to prove it, we shall need two technical lemmas - and our second necessary condition.

Lemma 4.1.2. Let $\Omega$ be a domain on the Riemann sphere and $z_{1}, z_{2}, \ldots$ points in $\Omega$. Suppose $f: \Omega \rightarrow(0,+\infty]$ is a function such that:

(i) $f$ is harmonic on $\Omega \backslash \bigcup_{i \geq 1}\left\{z_{i}\right\}$;

(ii) As $z \rightarrow z_{i}$, there exists some $m_{i}>0$ such that $f(z)=-m_{i} \log \left|z-z_{i}\right|+O(1)$ for every $i \geq 1$ (we say that $f$ has a logarithmic pole of order $m_{i}$ at $z_{i}$ );

(iii) $A s z \rightarrow \partial \Omega, f(z) \rightarrow 0$.

Then, $f$ can be decomposed as

$$
f(z)=\sum_{i \geq 1} m_{i} g_{\Omega}\left(z, z_{i}\right)
$$

where $g_{\Omega}\left(z, z_{i}\right)$ is the Green's function of $\Omega$ with pole at $z_{i}$.

Proof. Let

$$
h_{n}(z)=f(z)-\sum_{i=1}^{n} m_{i} g_{\Omega}\left(z, z_{i}\right) .
$$

We shall prove that the sequence $h_{n}$ converges to zero, and this will give us the conclusion. First, the fact that $g_{\Omega}$ is always non-negative implies that $h_{1} \geq h_{2} \geq h_{3} \geq \cdots$-i.e., $h_{n}$ is a monotonically decreasing sequence. Next, each $h_{n}$ is harmonic on $\Omega$ with the exception of logarithmic poles at $z_{i}$ for $i \geq n+1$ and goes to zero as $z$ approaches the boundary of $\Omega$. Therefore, we have that

$$
h_{1} \geq h_{2} \geq h_{3} \geq \cdots 0
$$

which means that the sequence $h_{n}$ converges point-wise to some function $h: \Omega \rightarrow \mathbb{R}$. Since it is the limit of a monotonic sequence of harmonic functions, $h$ is itself harmonic throughout $\Omega$, and it also satisfies $h(z) \rightarrow 0$ as $z \rightarrow \partial \Omega$. By the maximum modulus principle, $h \equiv 0$ and we are done.

Next, we would like to show that a symmetry of $J(R)$ must, in some way, respect the critical points of $R$. Indeed, let $C(R)$ be the set of critical points of $R$ together with their pre-images:

$$
C(R)=\bigcup_{n \geq 1} R^{-n}\{z \in \widehat{\mathbb{C}}: z \text { is a critical point of } R\}
$$

We call $C(R)$ the set of pre-critical points of $R$. We shall show that, under appropriate conditions on $R$, any symmetry of $J(R)$ must preserve $C(R)$. The first step is to show the invariance of the maximal entropy measure, which in turn relies on the following lemma.

Lemma 4.1.3. Let $R$ and $S$ be rational maps of degree $\geq 2$. Then, $J(R)=J(S) \Leftrightarrow J(R)$ is completely invariant under $S$ and $J(S)$ is completely invariant under $R$. In particular, $J(\sigma R)=$ $J(R)$ for any $\sigma \in \Sigma(R)$.

Proof. If $J(R)=J(S)$, the complete invariance of the Julia set follows immediately. To prove the converse, we recall that $J(S)$ is characterised as the minimal closed, completely invariant set with more than three points, and hence $J(R) \subset J(S)$. By the symmetry of the hypothesis, we also get that $J(S) \subset J(R)$ and so they are equal.

Now, consider $\sigma \in \Sigma(R)$. In order to conclude that $J(R)=J(\sigma R)$, we shall prove that $J(R)$ is invariant under $\sigma R$, and vice-versa. Firstly, since $\sigma$ is a symmetry of $J(R)$, we have by definition 
$\sigma[J(R)]=J(R)$ and so it is clear that $\sigma R[J(R)]=J(R)$. Also, by minimality of the Julia set, this implies that $J(\sigma R) \subset J(R)$.

All that is left is to prove that $R[J(\sigma R)]=R^{-1}[J(\sigma R)]=J(\sigma R)$, and we shall do it by contradiction. Suppose, then, that $J(\sigma R)$ is not backward invariant under $R$ (since $R$ is surjective, this is actually equivalent to assuming that $J(\sigma R)$ is not completely invariant). Thus, we can take $z \in J(\sigma R)$ such that $R^{-1}(z)$ contains at least one point - which, by an abuse of notation, we denote by $R^{-1}(z)$ - that is not in $J(\sigma R)$. In other words, $R^{-1}(z) \in F(\sigma R)$. However, we already know that $J(\sigma R)$ is a subset of $J(R)$, which is completely invariant under $R$ and therefore $R^{-1}(z) \in J(R)$. This will be the basis for a contradiction.

Since $R^{-1}(z)$ is in the Fatou set of $\sigma R$, it follows from the Arzelà-Ascoli theorem that $\left\{(\sigma R)^{k}\right\}_{k \geq 1}$ is equicontinuous there. Thus, for any $\epsilon>0$, we can take a neighbourhood $U$ of $z$ such that

$$
\operatorname{diam}\left[(\sigma R)^{k} R^{-1}(U)\right]<\epsilon \quad \text { for every } k \geq 1 .
$$

By taking a term from the family $(\sigma R)^{k}$, this becomes

$$
\operatorname{diam}\left[(\sigma R)^{k-1} \sigma(U)\right]<\epsilon \text { for every } k \geq 1 .
$$

Next, consider what the sequence of mappings $(\sigma R)^{k-1} \sigma$ means for the diameter. The function $\sigma$ is an isometry of the Riemann sphere; therefore, none of the $\sigma$ terms in this expression have any effect on the diameter. This means that the end result of $\operatorname{diam}\left[(\sigma R)^{k-1} \sigma(U)\right]$ is ultimately determined by the iteration of $R$. Also, since $z \in J(R)$ and $J(R)$ is completely invariant under both $\sigma$ and $R$, this means that $U$ is always mapped to a neighbourhood of some point in $J(R)$. Since $R$ eventually expands all neighbourhoods of points in the Julia set - by Montel's theorem -, we can conclude that $\operatorname{diam}\left[(\sigma R)^{k-1} \sigma(U)\right]$ should eventually grow larger than any value of $\epsilon$, and so we have reached a contradiction.

Lemma 4.1.3 gives us a way to fabricate another function with the same Julia set as $R$. If $R$ is a non-exceptional function without any parabolic or rotation domains, this implies (by Theorem 3.2.2) that $\mu_{R}=\mu_{\sigma R}$. This allows us to prove the following.

Proposition 4.1.2. If $R$ is a non-exceptional rational map with no parabolic or rotation domains and $\sigma \in \Sigma(R)$, then $\mu_{R}$ and $u_{R}$ are invariant under $\sigma$.

Proof. As discussed, $\mu_{R}=\mu_{\sigma R}$. By invariant of the maximal entropy measure,

$$
\mu_{R}=(\sigma R)_{*} \mu_{R}=\sigma_{*} R_{*} \mu_{R}=\sigma_{*} \mu_{R} .
$$

In other words, $\mu_{R}$ is $\sigma$-invariant. Now, the expression for $u_{R} \circ \sigma$ reads

$$
u_{R} \circ \sigma(z)=\int_{\widehat{\mathbb{C}}} \log \frac{1}{\rho[\sigma(z), w]} d \mu_{R}(w)
$$

and, as $\sigma$ is an isometry for the metric $\rho$,

$$
u_{R} \circ \sigma(z)=\int_{\widehat{\mathbb{C}}} \log \frac{1}{\rho\left[z, \sigma^{-1}(w)\right]} d \mu_{R}(w)=\int_{\widehat{\mathbb{C}}} \log \frac{1}{\rho(z, w)} d\left(\sigma^{-1}\right)_{*} \mu_{R}(w) .
$$

Since $\left(\sigma^{-1}\right)_{*} \mu_{R}=\mu_{R}$ (for $\sigma^{-1}$ is also a symmetry of $J(R)$ ), we recover the original expression for $u_{R}$ on the right-hand side of the equality above, and thus $\left(\sigma^{*} u_{R}\right)(z):=u_{R} \circ \sigma(z)=u_{R}(z)$.

Although $u_{R}$ is not trivial to compute, its invariance will give us a powerful tool for proving the invariance of $C(R)$ under symmetries. However, the proof relies heavily on another subharmonic function related to $R$ - the lift of its Green's function, $G_{\widehat{R}}$. 
Theorem 4.1.2. For a non-exceptional map $R$ without parabolic or rotation domains, any $\sigma \in \Sigma(R)$ preserves the pre-critical set.

Proof. By Theorem 3.3.1, we know that $u_{R}=-g_{R}+C$, where $g_{R}$ is the Green's function for $R$ and $C$ is some real constant - which we shall promptly ignore.

Thus, we have that $\log \|\cdot\|-G_{\widehat{R}}=\pi^{*} u_{R}$. By Proposition 4.1.2, $\sigma^{*} u_{R}=u_{R}$, so that $\log \|\cdot\|-G_{\widehat{R}}=$ $\pi^{*}\left(\sigma^{*} u_{R}\right)=(\sigma \pi)^{*} u_{R}$. The map $\sigma$, being an isometry of $\widehat{\mathbb{C}}$, lifts to a rotational isometry $\Sigma$ of $\mathbb{C}^{2}$, and therefore

$$
\log \|\cdot\|-G_{\widehat{R}}=\Sigma^{*}\left(\pi^{*} u_{R}\right)=\Sigma^{*}\left(\log \|\cdot\|-G_{\widehat{R}}\right)=\Sigma^{*} \log \|\cdot\|-\Sigma^{*} G_{\widehat{R}} .
$$

The fact that $\Sigma$ is a rotation of $\mathbb{C}^{2}$ means that the logarithm cancels out, so that $G_{\widehat{R}}=\Sigma^{*} G_{\widehat{R}}$. In particular, local minima of $G_{\widehat{R}}$ over $F(R)$ are mapped by $\Sigma$ onto local minima of $G_{\widehat{R}}$ over $F(R)$; we are going to prove that these points are exactly those with $D \widehat{R}=0$ (where $D \widehat{R}$ denotes the differential of $\widehat{R}$ ). Indeed, consider the sequence

$$
G_{n}:=\frac{1}{d^{n}} \log \left\|\widehat{R}^{n}\right\|
$$

Each of its terms is p.s.h. and smooth, and the sequence converges to $G_{\widehat{R}}$ uniformly over compact sets. Now, by the chain rule, the points with $D \widehat{R}^{n}=0$ are the minima of $G_{n}$; these, in turn, are exactly the $n$-th pre-images of critical points of $\widehat{R}$-i.e., those with $D \widehat{R}=0$. And, also by the chain rule, any local minimum of $G_{n}$ is also a local minimum of $G_{m}$ for any $m \geq n$. Since the convergence to $G_{\widehat{R}}$ is uniform over compact sets, this means that $G_{\widehat{R}}$ also has a local minimum at every such point. Conversely, every local minimum of $G_{\widehat{R}}$ comes from a converging sequence of local minima of $G_{n}$ for sufficiently large values of $n$.

We conclude, thus, that local minima of $G_{\widehat{R}}$ correspond to $C(\widehat{R})$, the pre-critical set of $\widehat{R}$. Since we already knew that local minima of $G_{\widehat{R}}$ are invariant under $\Sigma$, this implies that $\Sigma[C(\widehat{R})]=C(\widehat{R})$; finally, we come back to the Riemann sphere $-\Sigma$ pushes down to $\sigma, C(\widehat{R})$ pushes down to $C(R)$ and we conclude that $\sigma[C(R)]=C(R)$.

We are finally in a position to prove the first necessary condition mentioned before.

Proposition 4.1.3. Suppose $R$ is non-exceptional without any parabolic or rotation domains, and suppose that $\sigma \in \Sigma(R)$ fixes a superattracting point $z_{0}$ with local degree $m>1$. Then, $R \sigma=\sigma^{m} R$.

Proof. Consider the function

$$
f(z)=-\lim _{n \rightarrow \infty} \frac{1}{m^{n}} \log \left|\Phi\left[R^{n}(z)\right]\right|,
$$

where $\Phi: U \rightarrow B(0 ; r)$ is the Böttcher function for $z_{0}$. It is well defined throughout the immediate basin of attraction of $z_{0}$, denoted $F_{0}$, with logarithmic poles at the pre-images of $z_{0}$ with order given by the multiplicity of the pre-image. By Böttcher's theorem, $R$ maps level curves of $f$ onto level curves of $f$ - in fact, $f[R(z)]=m f(z)$. Furthermore, we can apply Lemma 4.1.2 to $f$ and write

$$
f(z)=\sum_{i \geq 0} m_{i} g_{F_{0}}\left(z, z_{i}\right)
$$

where we have enumerated the pre-images of $z_{0}$ in $F_{0}$ as $z_{0}, z_{1}, \ldots$ ( $z_{0}$ is a pre-image of itself). Now, we have:

$$
f[\sigma(z)]=\sum_{i \geq 0} m_{i} g_{F_{0}}\left[\sigma(z), z_{i}\right]=\sum_{j \geq 0} m_{j} g_{F_{0}}\left[\sigma(z), \sigma\left(z_{j}\right)\right],
$$

where in the last equality we have used Theorem 4.1.2 to ensure that $\sigma$ permutes the $z_{i}$, and permuted the indices accordingly. Next, the hypothesis that $\sigma$ is a symmetry of $J(R)$ fixing $z_{0}$ implies that $\sigma$ is a conformal mapping of $F_{0}$ onto itself, and - since Green's functions are preserved 
by conformal mappings - we conclude that $g_{F_{0}}\left[\sigma(z), \sigma\left(z_{j}\right)\right]=g_{F_{0}}\left(z, z_{j}\right)$ for every $j$. Therefore, $f \sigma(z)=f(z)$, which means that there exists a neighbourhood $V$ of $z_{0}$ that is forward-invariant under both $R$ and $\sigma$ and which is contained in $U$ - one need only define $V$ as any level curve of $f$ that is completely contained in $U$. Again by Böttcher's theorem (and the definition of $f, \Phi$ maps $V$ onto a circle $B(0 ; \delta), 0<\delta<r$, and conjugates $R$ to $z^{m}$.

Now, consider the functions $\widehat{\sigma}=\Phi \sigma \Phi^{-1}: B(0 ; \delta) \rightarrow B(0 ; \delta)$ and $\widehat{R}=\Phi R \Phi^{-1}: B(0 ; \delta) \rightarrow$ $B(0 ; \delta)$. Of course, we already know that $\widehat{R}(z)=z^{m}$; notice, then, that $\widehat{\sigma}$ is an automorphism of $B(0 ; \delta)$, and thus it is an isometry for the hyperbolic metric on $B(0 ; \delta)$. Since it also fixes 0 , it follows that it is of the form $\widehat{\sigma}(z)=e^{i \theta} z$ for some $\theta \in[0,2 \pi)$. Thus,

$$
\widehat{R} \widehat{\sigma}(z)=\left(e^{i \theta} z\right)^{m}=e^{i(m \theta)} z^{m}=\widehat{\sigma}^{m} \widehat{R}(z),
$$

and so $R \sigma=\sigma^{m} R$.

Proposition 4.1.3 is, in some sense, a partial converse statement to Proposition 4.1.1. The extra hypotheses, however, prevent us from promoting them to a characterisation of symmetries, and we know that they cannot fully be dropped. Take, for instance, the map

$$
R(z)=\frac{\left(z^{2}+1\right)^{2}}{4 z\left(z^{2}-1\right)}
$$

It is a Lattès map, so $J(R)=\widehat{\mathbb{C}}$ and thus $\Sigma(R)=\mathcal{I}(\widehat{\mathbb{C}})$. However, if we consider $\sigma(z)=e^{i \theta} z$, a straightforward calculation shows that

$$
R \sigma(z)=e^{i \theta} \frac{\left(z^{2}+e^{-2 i \theta}\right)^{2}}{4 z\left(z^{2}-e^{-2 i \theta}\right)}
$$

which is not $\sigma^{k} R$ for any value of $k$. It is worth mentioning that the maximal entropy measure $\mu_{R}$ is not invariant under $\mathcal{I}(\widehat{\mathbb{C}})$; if it were, it would be the Lebesgue measure on the Riemann sphere, but instead it is known to be absolutely continuous with respect to Lebesgue with the exception of finitely many singularities (see [Mil06b] in [HP06]).

We end this section with two other applications of Lemma 4.1.3. They are both, in a sense, related to Beardon's results. The first gives a sufficient condition for two rational maps to have the same Julia set - it is a relaxation of Julia's result that this happens if the two maps commute.

Proposition 4.1.4. If $R$ and $S$ are two rational maps of degree $\geq 2$ such that $S R=\sigma R S$ for some $\sigma \in \Sigma(R)$, then $J(R)=J(S)$.

Proof. We shall prove that the hypotheses imply that $F(R)$ is completely invariant under $S$ and vice-versa. Since both maps are surjective, it suffices to prove backward invariance, i.e. $S^{-1}[F(R)] \subset$ $F(R) \subset S^{-1}[F(R)]$.

Firstly, notice that, for all $k \geq 1$, we obtain by induction - the argument is analogous to the one used in Proposition 4.1.1 - that $S R^{k}=(\sigma R)^{k} S$. Now, let $M$ be a Lipschitz constant for $S$ in the chordal metric. For $z \in F(R)$, the definition of the Fatou set means that $\left\{R^{k}\right\}_{k \geq 1}$ is normal, and therefore equicontinuous by the Arzelá-Ascoli theorem, at $z$. As such, there exists for any $\epsilon>0$ a neighbourhood $U$ of $z$ such that $\operatorname{diam}\left[R^{k}(U)\right]<\epsilon / M$ for every $k \geq 1$. Now, by the induction formula,

$$
\operatorname{diam}\left[(\sigma R)^{k} S(U)\right]=\operatorname{diam}\left[S R^{k}(U)\right] \leq M \operatorname{diam}\left[R^{k}(U)\right]<\epsilon .
$$

This tells us that $\left\{(\sigma R)^{k}\right\}_{k \geq 1}$ is equicontinuous on $S(U)$, and thus $S(z) \in F(\sigma R)=F(R)$ by Lemma 4.1.3. Therefore, $S[F(R)] \subset F(R)$ and - since $F(R) \subset S^{-1} S[F(R)]$ - it follows that $F(R) \subset S^{-1}[F(R)]$.

Next, let $V=S^{-1}(U)$ for $U \subset F(R)$. As $F(\sigma R)=F(R)$ by Lemma 4.1.3, we can pick $U$ such 
that $\operatorname{diam}\left[(\sigma R)^{k}\right](U)<\epsilon$ for every $k \geq 1$ for an arbitrary choice of $\epsilon$. Then,

$$
\begin{aligned}
\operatorname{diam}\left[S R^{k}(V)\right] & =\operatorname{diam}\left\{S R^{k}\left[S^{-1}(U)\right]\right\} \\
& =\operatorname{diam}\left\{(\sigma R)^{k} S\left[S^{-1}(U)\right]\right\} \\
& =\operatorname{diam}\left[(\sigma R)^{k}(U)\right]<\epsilon,
\end{aligned}
$$

and so $\left\{S R^{k}(U)\right\}_{k \geq 1}$ is equicontinuous on $V$. Since $S$ is Lipschitz continuous, we have by the Arzelá-Ascoli theorem that $\left\{R^{k}\right\}_{k \geq 1}$ is normal on $V$. It follows that $S^{-1}[F(R)] \subset F(R)$, and so we conclude that $F(R)$ is backward invariant under $S$. Finally, this implies that $F(R)$ - and thus $J(R)$ - is completely invariant uder $S$.

For the remaining statement - the complete invariance of $F(S)$ under $R$-, we recall that $\Sigma(R)$ is a group; hence, $\sigma^{-1}$ is also a symmetry of the Julia set, and it satisfies $R S=\sigma^{-1} S R$. We can apply the argument above to conclude the converse, and by Lemma 4.1.3 we have $J(R)=J(S)$.

Our second application of Lemma 4.1.3 concerns a generalisation of Beardon's remark that Theorem 3.1.1 implies that most polynomials do not have any non-trivial symmetries. Our result, however, is stated in a more precise fashion thanks to Ye's work.

Theorem 4.1.3. Suppose $R \in \mathrm{Rat}_{d}, d \geq 3$, is non-exceptional without parabolic or rotation domains and has a non-trivial symmetry group. Then, $R$ is contained in a countable union of Zariskiclosed subsets of Rat . $_{\text {. }}$.

Proof. Take any non-trivial $\sigma \in \Sigma(R)$. By Lemma 4.1.3, the map $\sigma R$ has the same Julia set as $R$ - which, by Theorem 3.2.2, implies that $\mu_{R}=\mu_{\sigma R}$. But $\sigma R$ is not of the form $R^{n}$ for any natural $n$, and so the conclusion follows by Theorem 3.2.4.

\subsection{Applications}

In this section, we obtain concrete examples of symmetry groups for the maps

$$
R_{\lambda}(z)=z^{m}+\frac{\lambda}{z^{d}}
$$

with $m \geq 2, d \geq 1$ and $\lambda \in \mathbb{C}$. This family, called McMullen maps or singular perturbations, was already studied by McMullen [McM88, DEG ${ }^{+88}$ ], Devaney and co-workers [DLU05] and Hu and co-workers [HJM12, BDGS12]. These previous works have already expoited particular Möbius transformations preserving $J\left(R_{\lambda}\right)$, but here we provide a complete description of all the isometries of $\widehat{\mathbb{C}}$ that do so.

Theorem 4.2.1. The Julia set of $R_{\lambda}$ has the following symmetries:

(i) $z \mapsto e^{i \theta} z^{ \pm 1}$ for any $\theta \in[0,2 \pi)$, if $\lambda=0$;

(ii) $z \mapsto \mu z^{ \pm 1}$, where $\mu^{m+d}=1$, if $m=d$ and $|\lambda|=1$;

(iii) $z \mapsto \mu z$, where $\mu^{m+d}=1$, otherwise.

Proof. The case $\lambda=0$ reduces to $R_{0}(z)=z^{m}$, and the Julia set is a circle; hence, the conclusion follows from Theorem 4.1.1.

If $\lambda \neq 0$, any symmetry must either fix infinity or map it to another point. We start with the symmetries that fix it. These are, of course, a subgroup of $\Sigma\left(R_{\lambda}\right)$ made of isometries of the form $z \mapsto e^{i \theta} z$, and our task here is to ascertain the possible values of $\theta$. Let $\sigma(z)=\mu z$, where $\mu^{m+d}=1$. We shall prove that $\sigma \in \Sigma\left(R_{\lambda}\right)$. We have:

$$
R_{\lambda} \sigma(z)=\mu^{m} z^{m}+\frac{\lambda}{\mu^{d} z^{d}}=\mu^{m} z^{m}+\mu^{m} \frac{\lambda}{z^{d}}=\sigma^{m} R_{\lambda}(z)
$$


and so, by Proposition 4.1.1, $\sigma$ is a symmetry of $J\left(R_{\lambda}\right)$. On the other hand, any symmetry fixing infinity must, by Proposition 4.1.3, satisfy $R_{\lambda} \sigma=\sigma^{m} R_{\lambda}$. If $\sigma(z)=\nu z$ with $|\nu|=1$, then

$$
R_{\lambda} \sigma(z)=\nu^{m} z^{m}+\nu^{-d} \frac{\lambda}{z^{d}}=\nu^{m} z^{m}+\nu^{m} \frac{\lambda}{z^{d}}=\sigma^{m} R_{\lambda}(z),
$$

and thus $\nu^{-d}=\nu^{m}$ and $\nu^{m+d}=1$. Thus, the symmetries fixing infinity are a subgroup of $\Sigma\left(R_{\lambda}\right)$ isomorphic to $\mathbb{Z} /(m+d) \mathbb{Z}$.

Now, we consider any remaining symmetries. First, we invoke Theorem 4.1.1: since $\Sigma\left(R_{\lambda}\right)$ only admits certain structures, any symmetry group that properly contains $\left\{z \mapsto \nu z: \nu^{m+d}=1\right\}$ as a subgroup must also contain a symmetry of the form $\sigma(z)=\mu / z$ with $\mu^{m+d}=1$. What is left, thus, is to decide when it is one or the other.

Consider, then, that $\sigma \in \Sigma\left(R_{\lambda}\right)$ has the form $\sigma(z)=\mu / z$. Let $g_{0}(z, 0)$ and $g_{\infty}(z, \infty)$ denote the Green's functions for the connected components of $F\left(R_{\lambda}\right)$ containing 0 and $\infty$, respectively. Since conformal mappings send Green's functions to Green's functions, we have that $g_{\infty}[\sigma(z), \infty]=$ $g_{0}(z, 0)$ for $z$ in a neighbourhood of zero. At the same time, $R_{\lambda}$ maps this neighbourhood of 0 to a neighbourhood of infinity with multipllicity $d$, and so $g_{\infty}\left[R_{\lambda}(z), \infty\right]=d g_{0}(z, 0)$ by the uniqueness of the Green's function of a domain. Therefore, $g_{\infty}\left[R_{\lambda}(z), \infty\right]=d g_{\infty}[\sigma(z), \infty]$ and $d \log |\Phi \sigma(z)|=$ $\log \left|\Phi R_{\lambda}(z)\right|$, where $\Phi$ is the Böttcher functions for $R_{\lambda}$ at infinity; hence, by applying the series expansion for $\Phi$,

$$
\left[\frac{\mu}{z}+a_{0}+\cdots\right]^{d}=\alpha\left[z^{m}+\frac{\lambda}{z^{d}}+\cdots\right]
$$

where $|\alpha|=1$. Comparing the coefficients in the series expansion, we conclude that $m=d$ and, simultaneously, $\mu^{d}=\alpha \lambda$. Since $|\mu|=|\alpha|=1$, it follows that $|\lambda|=1$ and we are done.

Figures $4.1 \mathrm{a}$ and $4.2 \mathrm{a}$ illustrate this theorem. If $m=d=2$, the isometries $z \mapsto \mu z$, where $\mu^{4}=1$, are always a symmetry of $J\left(R_{\lambda}\right)$ (see Figures $4.1 \mathrm{~b}$ and $4.1 \mathrm{~d}$ ). If, in addition, $\lambda=0$ (red dot) or $|\lambda|=1$ (blue circle), then additional symmetries arise: in the former case, $J\left(R_{0}\right)$ is the unit circle, and so has all rotations as its symmetries as well as inversions with respect to the unit circle. In the latter, composing a rotation by a fourth root of unity with an inversion also yields a symmetry of $J\left(R_{\lambda}\right)$ (see Figure 4.1c). If, on the other hand, $m=2$ but $d=1$, the region $|\lambda|=1$ has nothing special with regards to symmetry. For any $\lambda \in \mathbb{C}^{*}$, the symmetry group consists of rotations by $2 \pi / 3$ and $4 \pi / 3$ radians as in Figures $4.2 \mathrm{~b}, 4.2 \mathrm{c}$ and $4.2 \mathrm{~d}$. For $\lambda=0$ (red dot) the Julia set is again the unit circle.

The figures show the connectedness locus - i.e., the values of $\lambda$ for which $J\left(R_{\lambda}\right)$ is connected - in order to emphasise one thing: the structure of the symmetry group has no regard for any topological changes to $J\left(R_{\lambda}\right)$. Indeed, while the structure of the Julia set changes drastically on the boundaries of the black region, the symmetry group "ignores" these changes and instead undergoes change as $|\lambda|=1$ for $m=d=2$, as in Figure 4.1a.

This sudden, quickly reversed change at $|\lambda|=1$ when $m=d$ can, in fact, be understood as the transversal movement of half-symmetries of $R_{\lambda}$ through $\mathcal{I}(\widehat{\mathbb{C}})$ in $\operatorname{PSL}(2, \mathbb{C})$. In [HJM12], the half-symmetries of a rational map $R$ were defined as the Möbius transformations $\gamma \in \operatorname{PSL}(2, \mathbb{C})$ satisfying $R \gamma=R$ - i.e., they permute the fibres of $R$. It is readily seen that these form a group $\mathcal{G}(R) \subset \operatorname{PSL}(2, \mathbb{C})$, and that $\gamma[J(R)]=J(R)$ for any $\gamma \in \mathcal{G}(R)$. It was proved by $\mathrm{Hu}$ and his co-workers that $\mathcal{G}(R)$ is always a finite group, and that it is conjugate to a group of isometries (see Theorem 4.1.1). For $R_{\lambda}$ and $m=d$, then, it is easy to check that $\gamma_{\lambda}(z)=\sqrt[m]{\lambda} / z$ is a half-symmetry depending on the value of $\lambda$. In other words, there is a continuous function $\lambda \mapsto \gamma_{\lambda}$ from $\mathbb{C}$ to $\operatorname{PSL}(2, \mathbb{C})$, and its image intersects $\mathcal{I}(\widehat{\mathbb{C}}) \subset \operatorname{PSL}(2, \mathbb{C})$ precisely as $|\lambda|=1-$ and then it moves away again, giving us the strange "bifurcation" of $\Sigma\left(R_{\lambda}\right)$ at the blue circle in Figure 4.1a. 


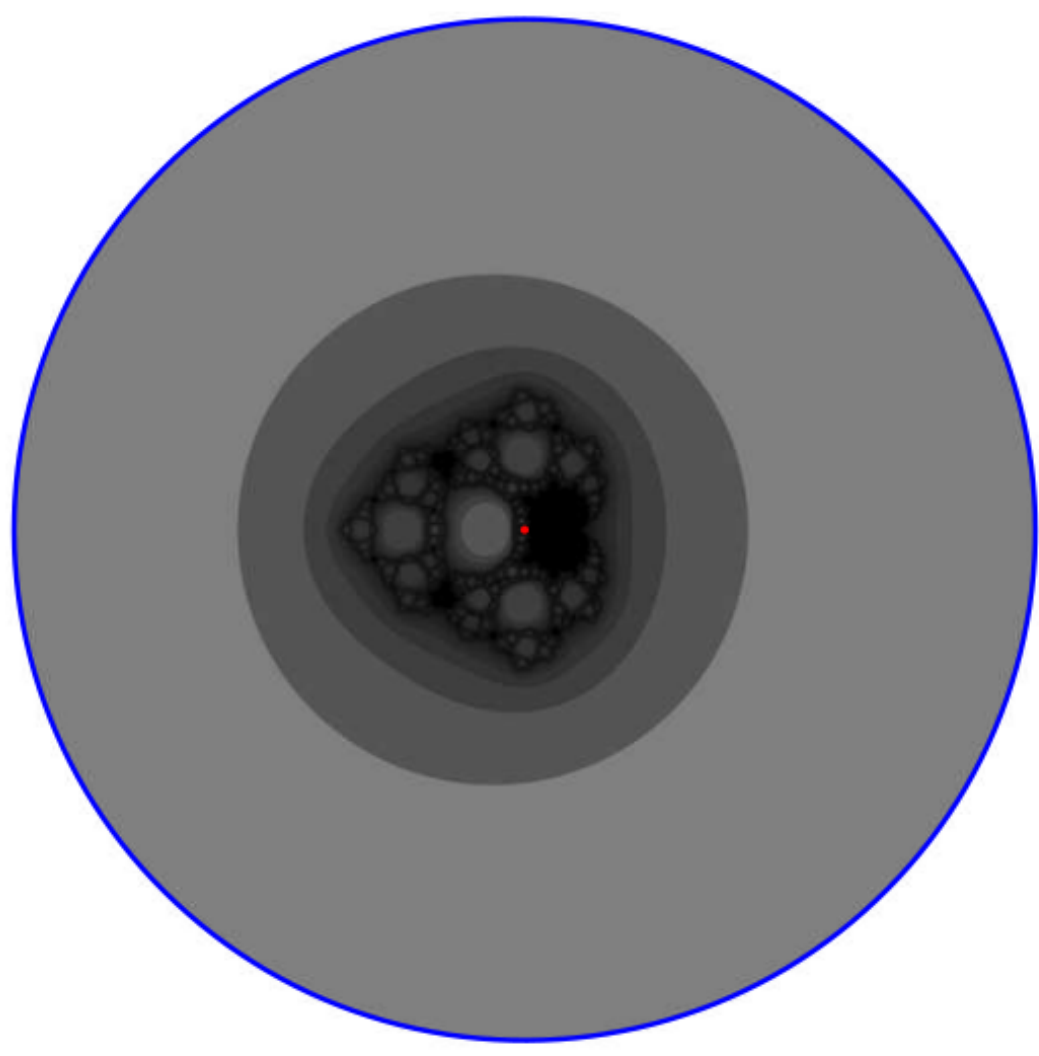

(a) Parameter plane for the family $z \mapsto z^{2}+\lambda / z^{2}$. We highlight the regions where $\Sigma\left(R_{\lambda}\right)$ is different.

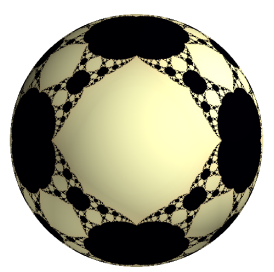

(b) $\lambda=0.1$

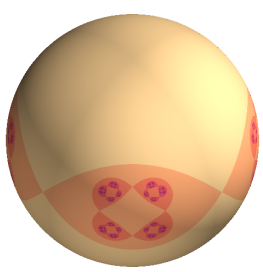

(c) $\lambda=1$

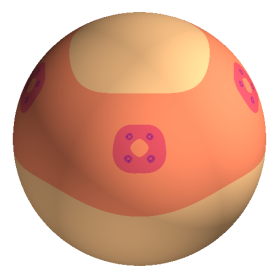

(d) $\lambda=10$

Figure 4.1: Above, the parameter plane for a family of singular perturbations with $m=d=2$. Below, Julia sets for particular values of $\lambda$. 


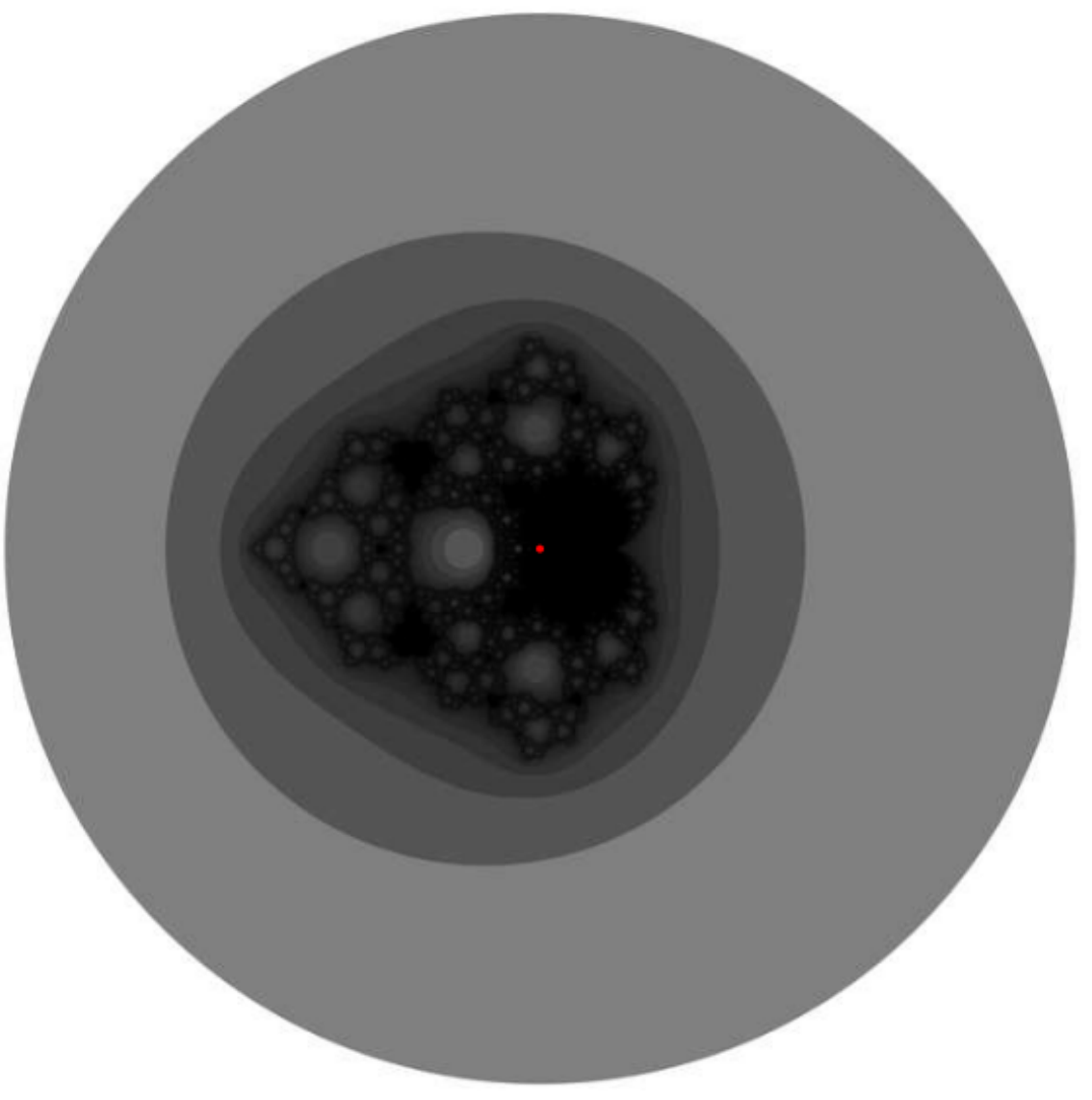

(a) Parameter plane for the family $z \mapsto z^{2}+\lambda / z$. We highlight the regions where $\Sigma\left(R_{\lambda}\right)$ is different.

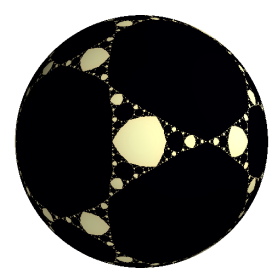

(b) $\lambda=0.1$

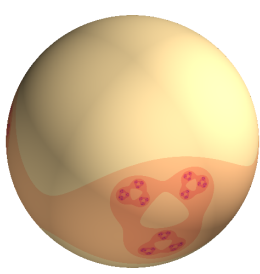

(c) $\lambda=1$

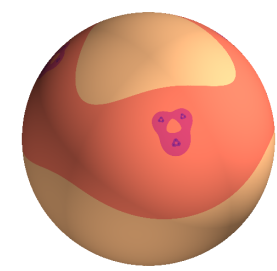

(d) $\lambda=10$

Figure 4.2: Above, the parameter plane for a family of singular perturbations with $m=2$ and $d=1$. Below, Julia sets for particular values of $\lambda$. 


\section{Chapter 5}

\section{Conclusions}

The symmetries of Julia sets of polynomials have shown themselves to be extremely useful tools for studying questions related to sharing Julia sets and maximal entropy measures. In this thesis, we applied this approach to rational maps and obtained new conditions for an isometry to be a symmetry of $J(R)$ for some rational map $R$, along with a complete description of the possible structures for the symmetry group $\Sigma(R)$. This was done by combining results and techniques from different areas of mathematics such as ergodic theory, Lie groups and potentials. We also proved that, in general, a rational functions's Julia set does not have any symmetries at all. Finally, we illustrated our results by describing the symmetries of Julia sets in the family $z \mapsto z^{m}+\lambda / z^{d}$ of singularly perturbed maps.

Of course, much remains to be done. Although we obtained necessary conditions and sufficient ones, a complete characterisation of symmetries for Julia sets remains elusive. Furthermore - as was pointed out to me by Mitsu Shishikura -, it would also be valuable to study the group of all Möbius transformations that preserve $J(R)$, of which $\Sigma(R)$ is a subgroup. 


\section{Bibliography}

[AB15] Marcos A. Alexandrino and Renato G. Bettiol. Lie Groups and Geometric Aspects of Isometric Actions. Springer, 2015. 9, 10, 23

[Bak06] Andrew Baker. Matrix Groups: An Introduction to Lie Group Theory. Springer, 3rd edition, 2006. 9

[BDGS12] Francis Bonahon, Robert L. Devaney, Frederick P. Gardiner and Dragomir Saric, editors. Conformal Dynamics and Hyperbolic Geometry. American Mathematical Society, 2012. 41,47

[BE87] Noel Baker and Alexander Eremenko. A problem on Julia sets. Ann. Acad. Sci. Fenn., 12:229-336, 1987. 1

[Bea90] Alan F. Beardon. Symmetries of Julia sets. Bulletin of the London Mathematical Society, 22:576-582, 1990. 1

[Bea91] Alan F. Beardon. Iteration of Rational Functions: Complex Analytic Dynamical Systems. Springer, 1991. 5

[Ber11] F. Berteloot. Bifurcation Currents in Holomorphic Families of Rational Maps, pages 1-93. Volume 1 of Bracci and Fornaess [BF11], 2011. 20, 32, 33

[BF11] F. Bracci and J. E. Fornaess, editors. Pluripotential Theory, volume 1. Springer, 2011. 20,46

[Bro65] Hans Brolin. Invariant sets under iteration of rational functions. Arkiv för Matematik, 6(2):103-144, 1965. 29

[Car12] T. K. Carne. Geometry and groups. https://www.dpmms.cam.ac.uk/ tkc/ GeometryandGroups/GeometryandGroups.pdf, 2012. 35

[DEG $\left.{ }^{+} 88\right]$ D. Drasin, C. J. Earle, F. W. Gehring, I. Kra and A. Marden, editors. Holomorphic Functions and Moduli I. Springer, 1988. 41, 47

[Dev03] Robert L. Devaney. An Introduction to Chaotic Dynamical Systems. CRC Press, 2nd edition, 2003. 4

[DLU05] Robert L. Devaney, D. M. Look and D. Uminsky. The escape trichotomy for singularly perturbed rational maps. Indiana University Mathematics Journal, 54:1621-1634, 2005. 41

[DM89] Peter Doyle and Curt McMullen. Solving the quintic by iteration. Acta Mathematica, 163:151-180, 1989. 36

[EvS10] Alexander Eremenko and Sebastian van Strien. Rational maps with real multipliers. Transactions of the American Mathematical Society, 363:6453-6463, 2010. 30, 36

[Fat20] Pierre Fatou. Sur les équations fonctionnelles. Bulletin de la Société Mathématique de France, 48:208-314, 1920. 36 
[Fat23] Pierre Fatou. Sur l'iteration analytique et les substitutions permutables. Journal de Mathématiques Pures et Apliquées, 3:1-50, 1923. 28

[FLM83] Alexandre Freire, Artur O. Lopes and Ricardo Mañé. An invariant measure for rational maps. Bulletin of the Brazilian Mathematical Society, 14(1):45-62, 1983. 29, 30

[HJM12] Jun Hu, Francisco G. Jimenez and Oleg Muzician. Rational maps with half-symmetries, Julia sets, and Multibrot sets in parameter planes, pages 119-137. Volume 1 of Bonahon et al. [BDGS12], 2012. 41, 42

[HN12] Joachim Hilgert and Karl-Hermann Neeb. Structure and Geometry of Lie Groups. Springer, 2012. 7, 9, 10

[HP06] PG Hjorth and CL Petersen, editors. Dynamics on the Riemann Sphere: A Bodil Banner Festschrift. European Mathematical Society, 2006. 40, 47

[Jul22] Gaston Julia. Memoire sur la permutabilité des fractions rationelles. Ann. Acad. Ecole Norm. Sup., 39:131-215, 1922. 1, 28

[Kli92] Maciej Klimek. Pluripotential Theory. Clarendon Press, 1992. 20, 22

[Lop86] Artur Oscar Lopes. Equilibrium measures for rational maps. Ergodic Theory and Dynamical Systems, 6:393-399, 1986. 30

[LP97] G. Levin and F. Przytycki. When do two rational functions have the same Julia set? Proceedings of the American Mathematical Society, 125:2179-2190, 1997. 30

[Lyu83] Mikhail Y. Lyubich. Entropy properties of rational endomorphisms of the Riemann sphere. Ergodic Theory and Dynamical Systems, 3:351-385, 1983. 29

[McM88] Curt McMullen. Automorphisms of rational maps, pages 31-60. Volume 1 of Drasin et al. [DEG+ 88], 1988. 41

[Mil06a] John Milnor. Dynamics in One Complex Variable. Princeton University Press, 3rd edition, 2006. 6, 36

[Mil06b] John Milnor. On Lattès Maps, pages 9-44. Volume 1 of Hjorth and Petersen [HP06], 2006. 40

[MNTU00] S. Morosawa, Y. Nishimura, M. Taniguchi and T. Ueda. Holomorphic Dynamics. Cambridge University Press, 2000. 4

[Oku05] Yusuke Okuyama. Complex dynamics, value distributions and potential theory. Annales Academiae Scientiarum Fennicae, 30:303-311, 2005. 33

[Ran95] Thomas Ransford. Potential Theory in the Complex Plane. Cambridge University Press, 1995. $10,12,16$

[Rit20] J. F. Ritt. On the iteration of rational functions. Transactions of the American Mathematical Society, 21:348-356, 1920. 28

[SS95] W. Schmidt and Norbert Steinmetz. The polynomials associated with a julia set. Bulletin of the London Mathematical Society, 27:239-241, 1995. 1, 23

[Tsu75] Masatsugu Tsuji. Potential Theory in Modern Function Theory. Chelsea Publishing Company, 2nd edition, 1975. 10

[vG] Onno van Gaans. Probability measures on metric spaces. https://www.math.leidenuniv. $\mathrm{nl} / \sim_{\text {vangaans/jancol1.pdf. } 15}$

[Ye15] Hexi Ye. Rational functions with identical measure of maximal entropy. Advances in Mathematics, 268:373-395, 2015. 1, 30 